\title{
Determinants of the benefit for consistent stimulus-response mappings in dual-task performance of four-choice tasks
}

\author{
Robert W. Proctor \\ Purdue University, West Lafayette, Indiana \\ AND \\ Kim-Phuong L. Vu \\ California State University, Long Beach, California
}

\begin{abstract}
Reaction times are shorter when the stimulus-response mappings for pairs of three-choice tasks are consistent (both corresponding or both mirrored) than when they are inconsistent. The benefit for consistent mirrored mappings is evident at the side positions for each task, for which the responses are crossed, but not at the middle position, for which the response is corresponding. In the present study, we report experiments in which we tested implications of an emergent mapping-choice account of the consistency benefit using pairs of four-choice tasks. This procedure allows crossed responses for all positions when the mapping is mirrored and use of mixed mappings for which one pair of stimuli and responses within a task has a corresponding assignment and the other a crossed assignment. Results showed that when a pure corresponding or pure mirrored mapping was used, there was a consistency benefit for both the middle and side positions. However, when the mixed mapping was introduced, the consistency benefit for that mapping depended on the overall complexity of the set of individual stimulus-response pairings for the combined tasks. A mapping choice between Tasks 1 and 2 is only one of several emergent processes that contribute to response-selection efficiency in dual-task contexts.
\end{abstract}

People have difficulty performing two tasks concurrently. For example, cell phone use produces deficits in driving and pedestrian behaviors that may have undesirable consequences (e.g., Strayer, Drews, \& Johnston, 2003). Likewise, a medical doctor may be distracted by having to answer a query from a colleague and may consequently fail to execute an intended action (e.g., Zhang, Patel, Johnson, \& Shortliffe, 2004). Laboratory studies have demonstrated that much of the difficulty in dual-task performance arises from central-processing demands imposed by response selection (e.g., Pashler, 1994). Similarly, costs of executing concurrent bimanual reaching movements of different amplitudes or directions to target stimuli are due primarily to limitations in responseselection processes (e.g., Albert, Weigelt, Hazeltine, \& Ivry, 2007). Therefore, consideration of factors that influence response-selection efficiency is vital to understanding and minimizing the difficulties associated with dualtask performance.

One factor that influences response-selection efficiency is the relation between stimulus-response (S-R) mappings for the two tasks. Duncan (1979) had participants perform pairs of three-choice reaction tasks to visual stimuli. For both tasks, the stimuli were vertical lines that could appear in any of three horizontal positions. The positions for Task 1 were located to the left of a fixation cross, and responses were made to the stimulus's location by pressing one of three keys with the ring, middle, or index finger of the left hand. The positions for Task 2 were to the right of the fixation cross, and responses were made with the same fingers on the right hand. In different trial blocks, participants performed using all pairings of two mappings - corresponding and mirrored - for each task. The corresponding mapping required a response at the location corresponding to the stimulus, whereas the mirrored mapping required opposite — or crossed — responses for the two side positions but the same response for the middle position.

Two of the Task 1-Task 2 mapping combinations were consistent (corresponding for both tasks or mirrored for both), and two were inconsistent (corresponding for Task 1 and mirrored for Task 2, or vice versa). Duncan (1979) examined performance only for the two side positions of each task, for which the responses with the mirrored mapping were crossed. The mean reaction times (RTs) for Task 1 (RT1) and for Task 2 (RT2) were shorter when the mappings were consistent than when they were inconsistent, a finding that we call the consistency benefit (alternatively, it is an inconsistency cost). Although RTs for consistent mirrored mappings were longer than

R.W. Proctor, proctor@psych.purdue.edu 
those for consistent corresponding mappings, they were still shorter than the RTs for either of the two conditions in which the mappings were inconsistent. Additionally, the consistency benefit did not interact systematically with stimulus onset asynchrony (SOA), which ranged from 50 to $450 \mathrm{msec}$ in his study.

Duncan (1979) attributed the consistency benefit to an emergent mapping choice for the conditions with inconsistent mappings. His idea was that more time is required to respond when the mappings for the two tasks are inconsistent, because a choice between mapping rules must be made prior to application of that rule. In support of this interpretation, Duncan (1979) noted that, when an error was made in the inconsistent mapping conditions, the error was not the response in the adjacent middle position but the response that would have been correct if the alternative mapping had been in effect. The implication is that, on the error trials, the wrong rule was selected in the initial choice, resulting in the incorrect response when it was subsequently applied.

We recently extended Duncan's (1979) findings for three-choice tasks in two major ways (Proctor \& Vu, 2008). First, we increased the SOA to a maximum of $1,000 \mathrm{msec}$ to determine whether the consistency benefit would be reduced at SOAs longer than those examined by Duncan (1979), as occurs for pairs of two-choice tasks (Ivry, Franz, Kingstone, \& Johnston, 1998; Vu \& Proctor, 2006). In agreement with Duncan's (1979) emergent mappingchoice account, the consistency benefit was equally evident for both corresponding and mirrored mappings at all SOAs. Second, we examined performance measures for the middle-position responses. Because the corresponding response is correct for the middle position regardless of the task mapping, middle-position responses provide an indication of whether, in inconsistent conditions, the mapping rule for each task (or hand) is selected as a whole. For the mirrored mapping, results did not show a consistency benefit at the middle positions, suggesting that no initial decision was being made as to whether the task mapping was corresponding or mirrored. This finding and others suggest that, for both the consistent mirrored and inconsistent conditions, participants first determined whether the stimulus position is in the middle or at a side. Only if the stimulus is determined to be in a side position is the step of selecting the mapping rule carried out.

Although the mirrored mapping did not show a consistency benefit at the middle positions, the corresponding mapping did. This benefit was of a size similar to that of the advantage that consistent corresponding mappings showed over consistent mirrored mappings at the side positions. This result implies that the consistency benefit for the corresponding mapping includes a facilitatory component that contributes to performance when all S-R pairings are corresponding. The facilitation is likely due to the fact that, in this case, the corresponding response to a stimulus can be executed without distinguishing whether the stimulus is from Task 1 or Task 2.

Mapping manipulations are limited for pairs of spatial three-choice tasks, because all possible task mappings other than pure corresponding require that a pair of po- sitions be crossed, with the remaining position being an exception for which the correct response is corresponding (as for the middle position with the mirrored mapping). This limitation can be avoided using four-choice tasks for which separate pairs of positions can be corresponding or crossed. With four-choice tasks, a mirrored mapping does not yield the corresponding response for any stimulus position and is composed of crossed assignments for the two middle and two side positions. Also, a mixed task mapping can be used that is itself inconsistent, with the assignment for the two middle locations being corresponding and that for the two side positions being crossed, or vice versa. These properties of four-choice tasks have been used to investigate response selection in single tasks (e.g., Duncan, 1977; Ehrenstein \& Proctor, 1998; Stoffels, 1996), but they have not been used to study the influence of mapping consistency in dual tasks.

In the present experiments, we exploited the properties of four-choice tasks to investigate mapping consistency benefits within and between tasks. Experiment 1 had the purpose of comparing performance with mirrored and corresponding mappings under conditions in which the mappings for the two tasks were consistent or inconsistent. Since the mirrored mapping requires crossed responses for the middle positions as well as for the side positions, the emergent mapping-choice account predicts that the middle-position responses should now show a consistency benefit similar to that shown by the side positions. Alternatively, because the side positions are more salient than the middle positions (Adam et al., 1998; Proctor, Vu, \& Pick, 2006), participants may still apply the "respond opposite" rule only to the side positions, as they would in three-choice tasks, which would restrict the benefit for consistent mirrored mappings to those positions. The experiment also examined whether the consistency benefit is evident at all SOAs, as is predicted by the emergent mapping-choice account. Such an examination is needed, because, although this predicted outcome has been obtained for three-choice tasks, it has not for twochoice tasks, which show a pattern of decreasing benefit as SOA increases (Ivry et al., 1998; Vu \& Proctor, 2006). Because two-choice tasks have an emergent perceptual feature at short SOAs (Vu \& Proctor, 2006) that the fourchoice tasks do not, finding that the consistency benefit occurs at all SOAs would provide converging evidence that the decreasing pattern observed in two-choice tasks is due to the emergent perceptual feature.

In Experiment 2, we used a mixed task mapping, for which the middle stimulus positions were assigned to corresponding responses and the side positions to crossed responses, instead of the mirrored mapping. In other respects, the experiment was similar to Experiment 1. When the task mappings were inconsistent, the S-R assignments were different for the side positions (corresponding for one task and crossed for the other) and the same for the middle positions (corresponding for both tasks), maintaining a structure similar to that for pairs of three-choice tasks. However, the mixed task mapping cannot be characterized by a single rule. If the consistency benefit arises from not having to choose between mapping rules, the 
mixed mapping should not show a consistency benefit, because the selection of mapping rules is still required. Moreover, the inconsistent mapping conditions could show a benefit over the consistent mixed mapping condition, because the global mapping across tasks is simpler, having six stimuli assigned to corresponding responses rather than just four.

If global mapping complexity is a factor that contributes to the relative ease with which two tasks can be performed together, a benefit for the consistent mixed mapping condition should be evident when the inconsistent conditions pair that mapping with a mirrored mapping, as was done in Experiment 3. To allow the middle position S-R assignment to be the same for each task, the mixed mapping of Experiment 3 required corresponding responses for the side positions and crossed responses for the middle positions. Thus, it was logically similar to that of Experiment 2, differing only in whether the middle or side positions were crossed. However, the inconsistent mapping conditions produced by combining the mixed and mirrored mappings for Tasks 1 and 2 were more complex overall, in that only two of the stimuli were assigned to corresponding responses. Thus, if the global complexity of the eight stimulus positions to responses is crucial, Experiment 3 should show a benefit for consistent mixed mappings. However, if the mappings for each task must follow a single rule in order to show a consistency benefit, there should again be no benefit for the consistent mixed mapping condition.

Response-response ( $\mathrm{R}-\mathrm{R}$ ) compatibility effects (differences in RTs for one task as a function of the position of the response for the other task) were analyzed for all experiments. Evidence indicates that the same physical response may be represented differently in different task contexts, leading to different types of R-R compatibility effects (Hazeltine, 2005; Schuch \& Koch, 2004). Analyses of such effects for three-choice tasks in Proctor and Vu's (2008) study provided some of the evidence that participants first made a decision as to whether the position was at a side or in the middle before - in the case of inconsistent mappings - determining which mapping rule to apply. Such a strategy is reasonable for pairs of three-choice tasks, because an initial decision that the stimulus position is in the middle specifies the response (i.e., the response is always the middle location, even when the task mappings are inconsistent). An initial middle-side decision is less likely to be made as part of the response-selection process for four-choice tasks, because the correct response is different for all stimulus positions when one task mapping is corresponding and the other is mirrored. However, if participants represent the mirrored mapping as composed of middle and side subsets, each with crossed responses, they may still make a middle-side determination. If so, $\mathrm{R}-\mathrm{R}$ compatibility effects should occur on the basis of the middle-side distinction and not on the basis of the specific responses within the middle and side pairs.

The types of errors committed were also analyzed. As has been noted, if the wrong mapping rule is applied to a stimulus, alternative-rule errors should predominate. For the side positions, this means that the errors should mainly be at nonadjacent positions. In contrast, if the cor- rect mapping is applied to the stimulus and errors arise from confusion about which finger to move, adjacent errors should occur even if they are not in agreement with the alternative rule.

\section{EXPERIMENT 1}

In Experiment 1, we examined all pairings of corresponding and mirrored mappings for Tasks 1 and 2, as was done in prior studies of the consistency benefit using three-choice tasks (Duncan, 1979; Proctor \& Vu, 2008). However, with four-choice tasks, there is no stimulus position for which the response is the same for both the mirrored and the corresponding mappings. Additionally, the mirrored mapping is more complex than one in a threechoice task, because there are two pairs of locations for which the assigned responses are crossed, rather than only one pair. Because each task mapping can be characterized by a single rule, the emergent mapping-choice account predicts a consistency benefit for both the mirrored and the corresponding mappings.

A unique prediction of the account for the four-choice task paradigm is that the mirrored mapping will show a consistency benefit for the middle positions that it did not for that in three-choice tasks (Proctor \& Vu, 2008). This benefit is predicted because the "respond opposite" rule applies to the middle positions as well as to the side positions. Finally, the consistency benefit for the mirrored and corresponding mappings should be evident at all SOAs, as it is with three-choice tasks, because there is no emergent perceptual feature that maps systematically onto responses for the two tasks, as there is with two-choice tasks.

\section{Method}

\section{Participants}

Sixteen undergraduate students from Purdue University participated for credit toward an introductory psychology course requirement. All had normal or corrected-to-normal vision.

\section{Apparatus and Stimuli}

Stimuli were presented on personal computers, with 14-in. VGA color monitors. Micro Experimental Laboratory (MEL v. 2.01) was used to program the experiment. The participant sat directly in front of the monitor, at a distance of approximately $50 \mathrm{~cm}$. A crosshair $\left(0.5^{\circ} \times 0.5^{\circ}\right)$ located in the center of the screen served as a fixation point. Four outline boxes $(1.8 \times 3.4 \mathrm{~cm})$ were presented to the left of fixation, and four to the right of fixation, to serve as placeholders for possible positions in which the imperative stimuli could appear (see Figure 1). The boundaries of the boxes were separated by $1.0 \mathrm{~cm}$; the four boxes for Task 1 were outlined in green, and the four for Task 2 , in red. The imperative stimulus was a solid white circle (MEL color code 15; approximately $1.0 \mathrm{~cm}\left[1.14^{\circ}\right]$ in diameter) presented in the center of one of the boxes for each task.

For each trial, the fixation cross and the eight placeholder boxes were presented by themselves for $500 \mathrm{msec}$. Then, the imperative stimulus for Task 1 appeared inside one of the four boxes to the left of fixation. After an SOA of 50,150, 400, or 1,000 msec, the imperative stimulus for Task 2 appeared inside one of the four boxes to the right of fixation. Both Stimulus 1 (S1) and Stimulus 2 (S2) remained on the screen until both Response 1 (R1) and Response 2 (R2) had been made, at which time the whole screen went blank. The intertrial interval was $1 \mathrm{sec}$.

Responses were made on a QWERTY keyboard. Responses for Task 1 were made by pressing the " $z$ " key with the left pinky, the " $\mathrm{x}$ " 


\section{Experiment 1: Corresponding and Mirrored Mappings}

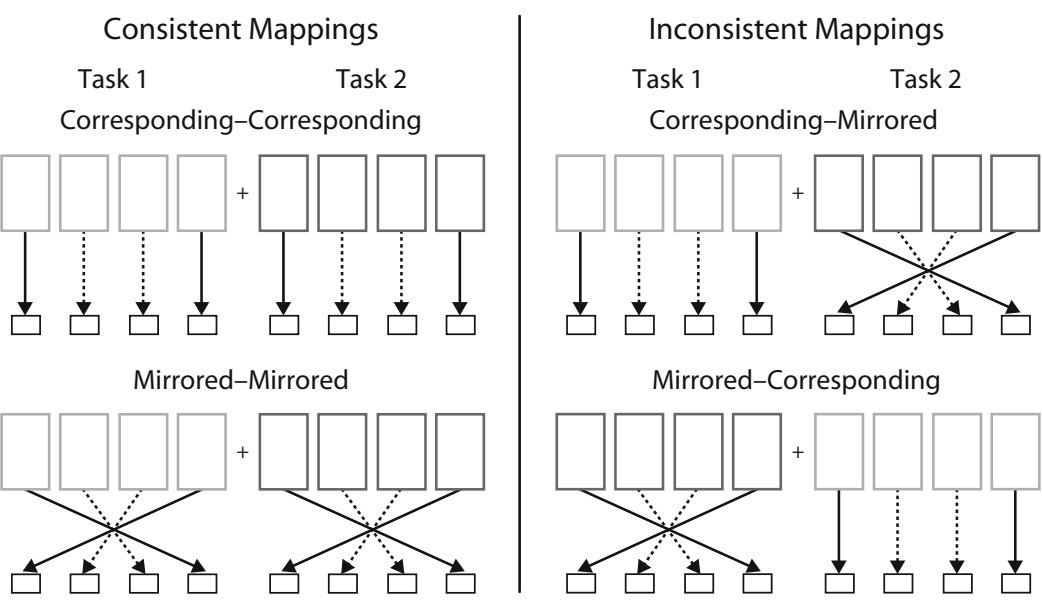

Figure 1. Illustration of the combinations of corresponding and mirrored mappings used in Experiment 1. The left column shows consistent mappings for the two tasks, and the right column shows inconsistent mappings.

key with the left ring finger, the "c" key with the left middle finger, and the " $v$ " key with the left index finger. Responses for Task 2 were made by pressing the " $m$ " key with the right index finger, the "," key with the right middle finger, the "." key with the right ring finger, and the "/" key with the right pinky.

\section{Procedure}

Participants were tested in a single session. In separate blocks, they performed all four Task 1-Task 2 mapping combinations (see Figure 1). The order in which the conditions were performed was counterbalanced across participants using a Latin square design. For the corresponding mapping, participants were instructed to press the key corresponding to the stimulus location. For the mirrored mapping, the mirror-opposite key was to be pressed for each stimulus location. Participants were told that the stimuli for Task 1 could appear in one of the four leftmost (green) boxes and that they were then to respond with their left hand, and they were told that the stimuli for Task 2 could appear in one of the four rightmost (red) boxes and that they were then to respond with their right hand. Participants were instructed to respond to both tasks as quickly and accurately as possible, performing Task 1 without waiting for the Task 2 stimulus to appear.

For each condition, 16 practice trials, for which feedback regarding accuracy on both tasks was given after each trial, preceded the experimental block. This block consisted of 192 trials, with no accuracy feedback, with 48 of the stimuli for each task occurring in each of the four stimulus positions in a random order. The pairing of a stimulus for one task with each of the 4 stimuli for the other task was randomized such that there was an average of 12 trials for each $\mathrm{S} 1-\mathrm{S} 2$ combination. The experimenter remained in the room for the entire experiment. RTs were measured from stimulus onset for each task to the depression of an appropriate response key.

\section{RTs and Percent Errors}

\section{Results}

Trials with RTs less than 200 or more than $4,000 \mathrm{msec}$ $(<1 \%$ in all experiments) were omitted as outliers. Mean RTs and percent errors (PEs) for each task were submitted to 2 (Task 1 mapping, corresponding or opposite) $\times 2$ (Task 2 mapping, corresponding or opposite) $\times 4$ (SOA, $50,150,400$, or $1,000 \mathrm{msec}) \times 2$ (position, middle or side) repeated measures ANOVAs. The reported effects for the primary analyses, as well as for all others, were significant with the Huynh-Feldt correction for violations of sphericity, except where noted.

Task 1. RT1 showed main effects of Task 1 mapping $\left[F(1,15)=14.94, M S_{\mathrm{e}}=74,917, p=.002\right]$ and of Task 2 mapping $\left[F(1,15)=5.02, M S_{\mathrm{e}}=42,165, p=.041\right]$. For both task mappings, RT1 was shorter when the mapping was corresponding $(M \mathrm{~s}=796$ and $823 \mathrm{msec}$, for Task 1 and Task 2 mappings, respectively) than when it was mirrored $(M \mathrm{~s}=890$ and $863 \mathrm{msec}$, respectively). Task 1 mapping interacted with Task 2 mapping $[F(1,15)=50.10$, $\left.M S_{\mathrm{e}}=78,845, p<.001\right]$; RT1 was shorter when the mappings were consistent $(M=755 \mathrm{msec})$ than when they were inconsistent $(M=931 \mathrm{msec})$.

There was a main effect of SOA $\left[F(3,45)=5.64, M S_{\mathrm{e}}=\right.$ $29,865, p<.025]$ : RT1 decreased as SOA increased $(M \mathrm{~s}=$ $876,870,825$, and $801 \mathrm{msec}$ ). The three-way interaction of SOA with Task 1 and Task 2 mappings was not significant $(F<1$; see Figure $2 \mathrm{~A})$, nor were any of the other terms, including all that involved middle versus side position.

The mean PE for Task 1 (PE1) showed main effects of Task 1 mapping $\left[F(1,15)=10.50, M S_{\mathrm{e}}=31.22, p=\right.$ $.005]$ and of Task 2 mapping $\left[F(1,15)=6.62, M S_{\mathrm{e}}=\right.$ $38.34, p=.021$ ] (see Table 1). For Task 1 mapping, PE1 was higher when the mapping was corresponding $(M=$ $3.64 \%)$ than when it was mirrored $(M=2.04 \%)$, mainly because the error rate was high for the correspondingmirrored condition. For Task 2 mapping, PE1 was lower when the mapping was corresponding $(M=2.14 \%)$ than when it was mirrored $(M=3.55 \%)$. Task 1 mapping interacted with Task 2 mapping $\left[F(1,15)=24.38, M S_{\mathrm{e}}=\right.$ 47.60, $p<.001$ ]; PE1 was lower when the mappings for Task 1 and Task 2 were consistent $(M=1.34 \%)$ than when they were inconsistent $(M=4.35 \%)$. The only significant term involving position (middle or side) was the interaction with Task 1 mapping $\left[F(1,15)=6.33, M S_{\mathrm{e}}=19.37\right.$, $p=.024]$. The Task 1 mapping effect was smaller for the side-position responses $(M=0.62 \%)$ than for the middleposition responses $(M=2.58 \%)$. 

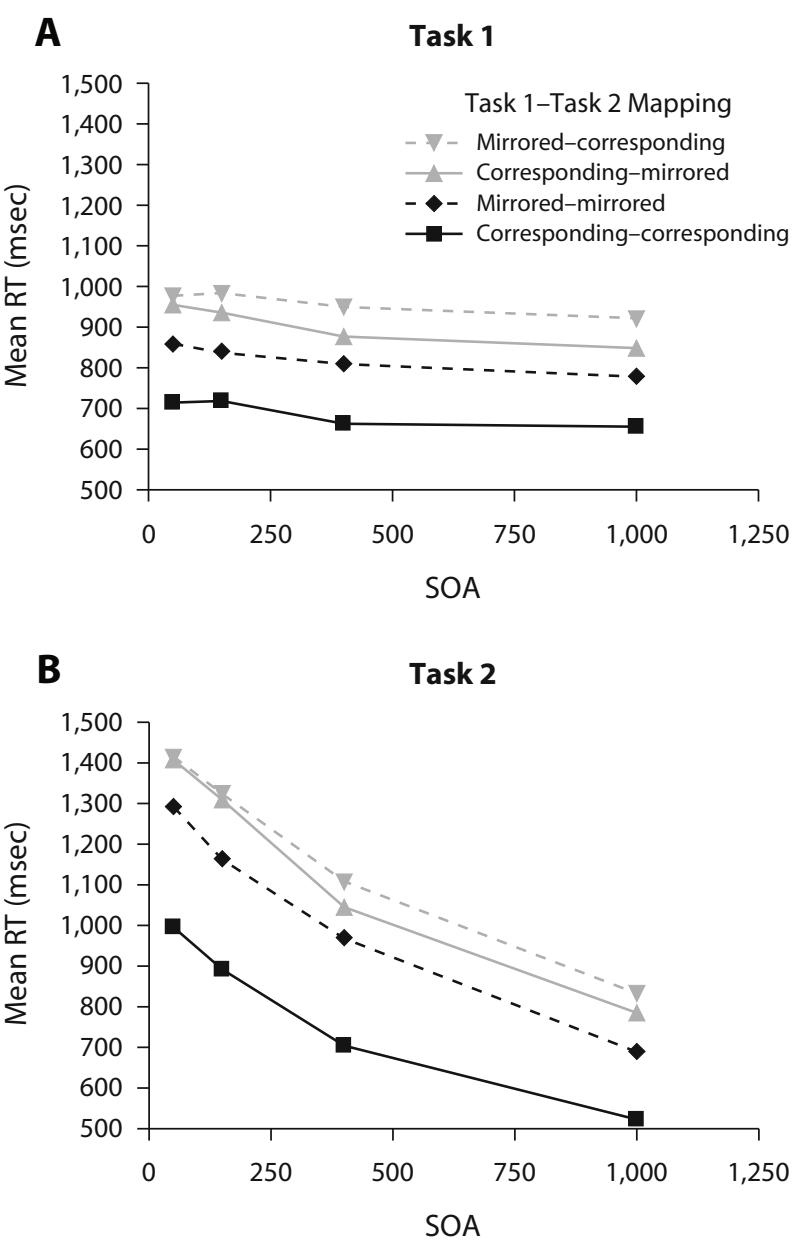

Figure 2. Mean reaction times (RTs) for Task 1 (A) and Task 2 (B) in milliseconds as a function of Task 1-Task 2 mapping and stimulus onset asynchrony (SOA) in Experiment 1.

Task 2. RT2 showed effects of Task 1 mapping $\left[F(1,15)=13.31, M S_{\mathrm{e}}=192,403, p=.002\right]$ and of Task 2 mapping $\left[F(1,15)=13.44, M S_{\mathrm{e}}=112,121, p=.002\right]$. For both task mappings, RT2 was shorter when the mapping was corresponding $(\mathrm{Ms}=958$ and $974 \mathrm{msec}$, for Task 1 and Task 2 mapping, respectively) than when it was mir-

Table 1

Mean Percentages of Error for Task 1 and Task 2 As a Function of Task 1-Task 2 Mapping and Stimulus Onset Asynchrony (SOA, in Milliseconds) for Experiment 1

\begin{tabular}{lcccr}
\hline & \multicolumn{4}{c}{ SOA } \\
\cline { 2 - 5 } \multicolumn{1}{c}{ Task 1-Task 2 Mapping } & 50 & 150 & 400 & 1,000 \\
\hline & Task 1 & & & \\
Corresponding-corresponding & 1.69 & 0.92 & 1.69 & 1.43 \\
Corresponding-mirrored & 4.19 & 6.39 & 6.43 & 6.39 \\
Mirrored-corresponding & 2.76 & 2.09 & 2.49 & 4.06 \\
Mirrored-mirrored & 1.71 & 0.52 & 1.17 & 1.57 \\
& Task 2 & & & \\
Corresponding-corresponding & 6.77 & 4.69 & 5.08 & 3.52 \\
Corresponding-mirrored & 6.66 & 4.61 & 5.15 & 5.91 \\
Mirrored-corresponding & 7.17 & 9.55 & 7.54 & 10.30 \\
Mirrored-mirrored & 4.18 & 3.53 & 4.31 & 3.01 \\
\hline
\end{tabular}

rored $(M \mathrm{~s}=1,100$ and 1,083 msec, respectively). Task 1 mapping again interacted with Task 2 mapping $[F(1,15)=$ 53.17, $\left.M S_{\mathrm{e}}=148,724, p<.001\right]$, with RT2 being shorter when the mappings were consistent $(M=904 \mathrm{msec})$ than when they were inconsistent $(M=1,154 \mathrm{msec})$.

The main effect of SOA was also significant $[F(3,45)=$ 251.22, $\left.M S_{\mathrm{e}}=32,433, p<.001\right]$ : There was a psychological refractory period (PRP) effect (Pashler, 1994), in which RT2 was longer at short SOAs than at longer ones $(M \mathrm{~s}=1,277,1,173,957$, and $708 \mathrm{msec}$ for SOAs from short to long). SOA interacted with both Task 1 mapping $\left[F(3,45)=3.57, M S_{\mathrm{e}}=5,184, p<.025\right]$ and Task 2 mapping $\left[F(3,45)=5.66, M S_{\mathrm{e}}=7,869, p=.002\right]$, and these interactions were qualified by a three-way interaction of the variables $\left[F(3,45)=4.17, M S_{\mathrm{e}}=10,053, p<.015\right]$ (see Figure $2 \mathrm{~B}$ ). The latter interaction was primarily due to the corresponding-corresponding condition's showing less decrease across SOAs than the other three conditions did. When the corresponding-corresponding condition was removed and the other three conditions were compared, the interaction with SOA was no longer significant $[F(6,90)=1.23, p=.30]$, but the main effect was $[F(2,30)=4.86, p=.015]$. The only significant term involving middle versus side position on RT2 was the main effect $\left[F(1,15)=16.24, M S_{\mathrm{e}}=20,701, p<.001\right]$, for which side-position responses $(M=1,004 \mathrm{msec})$ were faster than middle-position ones $(M=1,055 \mathrm{msec})$.

The mean PE for Task 2 (PE2) showed a main effect of Task 2 mapping (see Table 1) $\left[F(1,15)=4.65, M S_{\mathrm{e}}=\right.$ $127.44, p=.048]$ : PE2 was higher when the mapping was corresponding $(M=6.83 \%)$ than when it was mirrored $(M=4.68 \%)$, mainly because of a high error rate for the mirrored-corresponding condition $(M=8.65 \%)$. Task 1 mapping interacted with Task 2 mapping $[F(1,15)=$ 13.36, $\left.M S_{\mathrm{e}}=71.95, p=.002\right]$; PE2 was lower when the Task 1 and Task 2 mappings were consistent $(M=$ $4.39 \%)$ than when they were inconsistent $(M=7.13 \%)$. The three-way interaction with SOA was also significant $\left[F(3,45)=3.44, M S_{\mathrm{e}}=22.61, p=.024\right]$. The latter interaction was primarily due to PE2s decreasing across SOAs for the corresponding-corresponding condition but increasing for the mirrored-mirrored condition and being relatively stable for the other two conditions. Two terms involving position were significant: the main effect $\left[F(1,15)=20.70, M S_{\mathrm{e}}=38.15, p<.001\right]$ and the interaction with Task 2 mapping $\left[F(1,15)=5.86, M S_{\mathrm{e}}=25.03\right.$, $p=.029]$. Side-position responses showed fewer errors $(M=4.52 \%)$ and a smaller Task 2 mapping effect $(M=$ $1.08 \%)$ than did the middle-position ones $(M=7.00 \%$ and $3.22 \%$ ).

\section{R-R Compatibility}

R-R compatibility effects are typically most pronounced at short SOAs (e.g., Lien \& Proctor, 2000). However, since our main concern was with how the effects differed between middle and side positions and as a function of mapping consistency, we collapsed across SOA for these analyses. Responses for the task for which performance was being analyzed were classified according to their relations to the response for the other task: That 
relation could be (1) same relative position (compatible), (2) mirror-opposite position (which is also anatomically corresponding), or (3) one of the two remaining positions (incompatible). Separate ANOVAs for RT1 and RT2 as a function of position and R-R compatibility (compatible, mirror opposite, or incompatible) were performed. The mean data are shown in Figure 3, and the detailed analyses are described in Appendix A.

The R-R compatibility analysis showed little evidence either that having Task 1 and Task 2 responses in corresponding locations was beneficial or that participants made an initial middle-side decision for conditions in which at least one task mapping was mirrored. RT1 showed no significant R-R compatibility effect. RT2 showed a benefit for the side-response positions when R1 was in either the compatible or the mirror-opposite position, but this was primarily for the conditions with consistent task mappings. For those conditions, the R-R compatibility effect for RT2 was on the side positions and not on the specific response (or stimulus) position. Restriction of this effect to the side $\mathrm{R} 2$ positions is likely a consequence of those positions being more salient than the middle po-

\section{A}

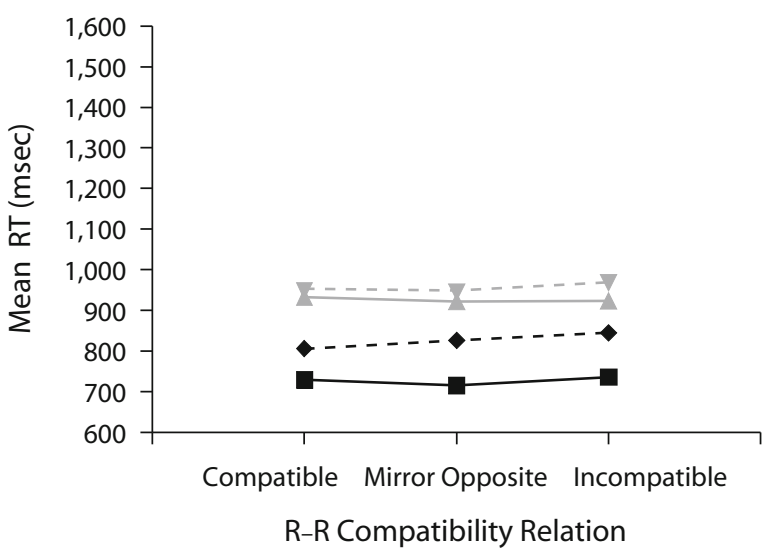

C

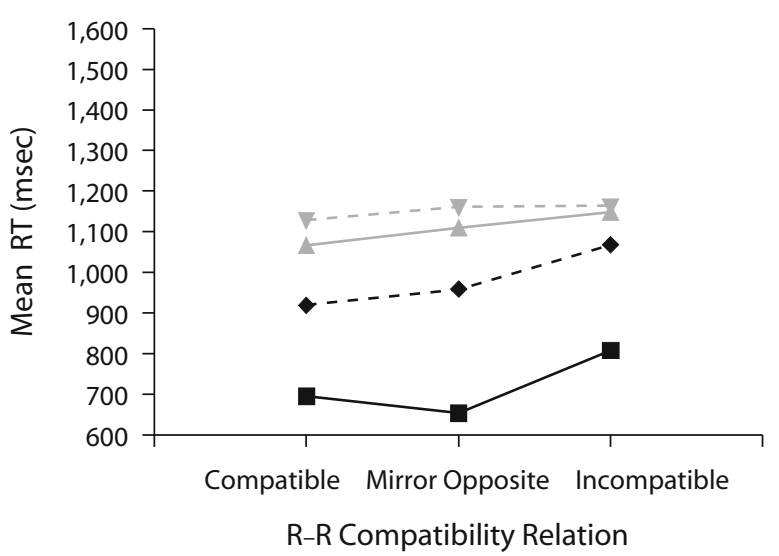

sitions were (e.g., Adam et al., 1998). Taken together, the pattern of effects indicates that $\mathrm{R}-\mathrm{R}$ compatibility is not the source of the mapping consistency benefit.

\section{Error Types}

For the consistent mapping conditions, an error could involve making the response in the mirror-opposite position, an adjacent position (nonmirror opposite for middle responses), or the other position, which is neither adjacent to the correct response nor opposite to it. For the inconsistent mapping conditions, the mirror-opposite response is also the one that would be correct for the alternative mapping rule. The frequencies of each error type are shown in Figure 4. We provide a verbal description of the major results here, and the analyses are included in Appendix B.

On the whole, fewer errors occurred for Task 1 than for Task 2. For both tasks, the error rate was higher when the mappings were inconsistent than when they were consistent. For the consistent conditions, most errors were to make the response (corresponding or opposite) that would be correct for the alternative mapping rule or the response at an adjacent position that did not match the alternative

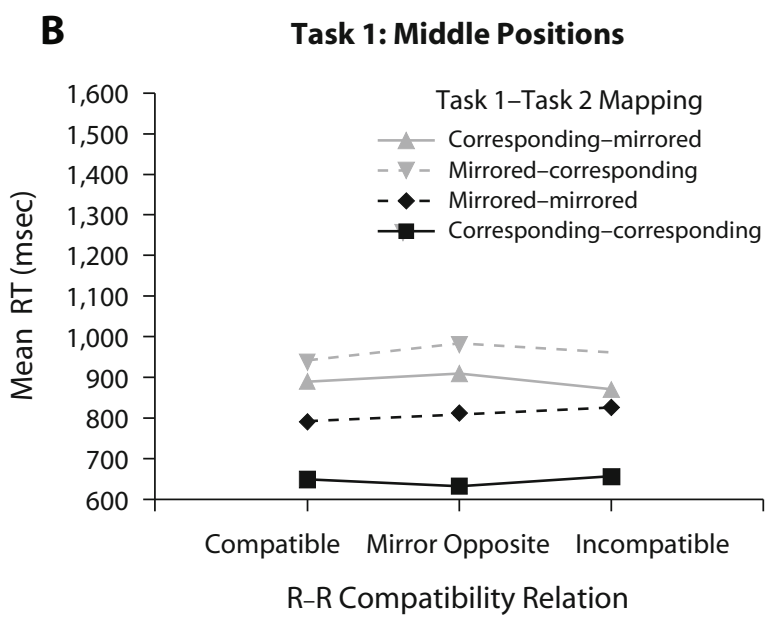

D

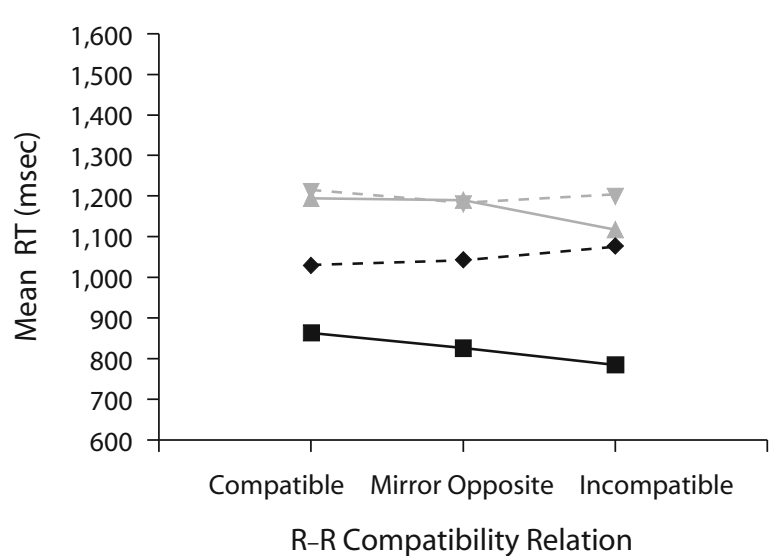

Figure 3. Mean reaction times (RTs) for Task 1 (A and B) and Task 2 (C and D) in milliseconds for the side (A and C) and middle (B and D) stimulus positions as a function of response-response ( $R-R)$ compatibility and Task 1-Task 2 mappings in Experiment 1. 
A

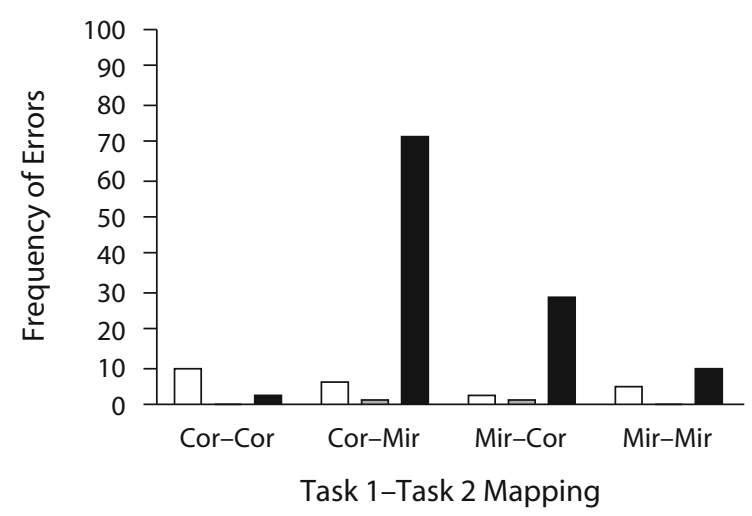

C

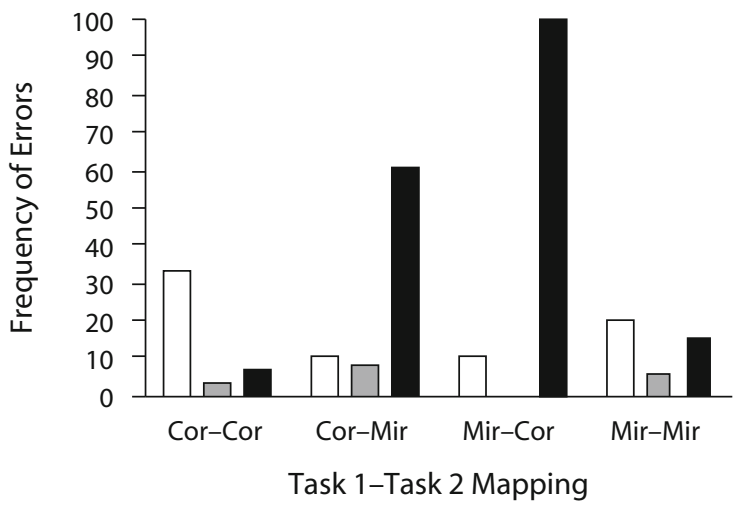

B

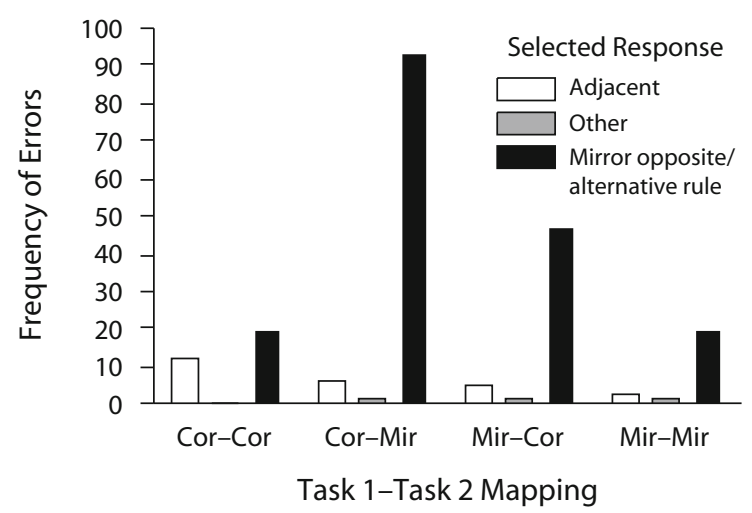

D

Task 2: Middle Responses

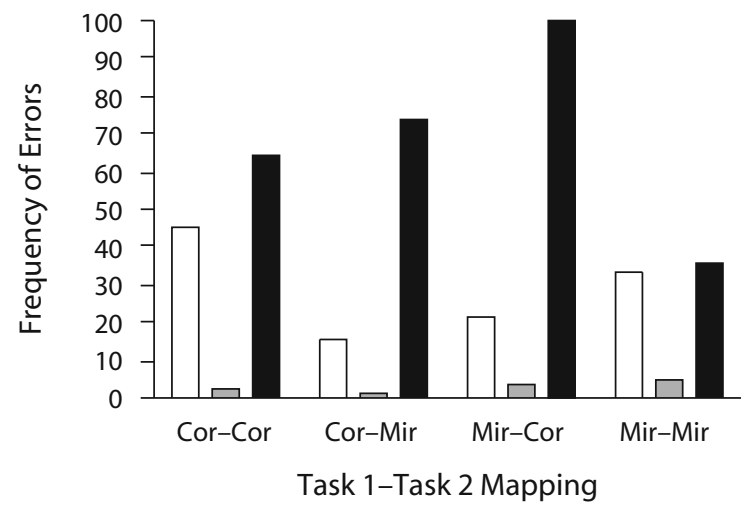

Figure 4. Frequencies of errors for Task 1 (A and B) and Task 2 (C and D) as a function of Task 1-Task 2 mapping and selected response in Experiment 1. Cor, corresponding mapping; Mir, mirrored mapping.

rule. More important, for the inconsistent conditions, for both the middle and side positions, the most frequent errors were the responses that would have been correct if the mapping for one task were applied incorrectly to the other. This pattern of results for inconsistent task mappings is in agreement with the emergent mapping-choice account.

\section{Discussion}

A consistency benefit was apparent for the four-choice tasks. In this case, where all stimulus positions required a different response for the mirrored mapping than for the corresponding mapping, the benefit was evident for the mirrored-mirrored condition at the middle positions as well as at the side positions. The consistency benefit was larger for the corresponding-corresponding condition than for the mirrored-mirrored condition, indicating facilitation that results from all individual S-R assignments when both tasks are corresponding. The three-way interaction of the task mappings and SOA for RT1 showed an $F$ ratio of less than 1, which is particularly critical for the emergent mapping-choice account, because the duration of the SOA is not known at the time that S1 appears. For RT2, the three-way interaction involving SOA was significant. However, even in this case, where a long SOA could possibly allow time to prepare for the Task 2 mapping, the interaction was due mainly to the correspondingcorresponding condition's deviation from the other three and did not approach statistical significance when that condition was removed. Moreover, the PE2 data showed the opposite pattern: The corresponding-corresponding condition benefitted more from an increase in SOA than did the other conditions. Thus, the results are in agreement with those from Proctor and Vu's (2008) study of threechoice tasks; they show a benefit for consistent mappings that is not affected much by SOA, as was predicted by the emergent mapping-choice account. That most errors in the inconsistent conditions were alternative-rule errors is also in agreement with that account.

\section{EXPERIMENT 2}

For three-choice tasks, the middle positions show no consistency benefit for the mirrored mapping and a reduced benefit for the corresponding mapping, which reflects facilitation due to all S-R pairings for the two tasks being corresponding (Proctor $\& \mathrm{Vu}, 2008$ ). The absence of a consistency benefit due to the emergent mapping choice is likely a consequence of the middle position's requiring the corresponding response for all task mapping combinations. In Experiment 1, we provided evidence in support of 
this interpretation by showing that, for four-choice tasks, the two middle positions yielded mapping consistency benefits that did not differ reliably from those exhibited by the two side positions. Thus, application of a consistent "respond opposite" mapping rule is not restricted to the more salient side positions of the linear arrangements.

In Experiment 2, we examined a task mapping for which the opposite outcome is predicted: no consistency benefit at either the middle or the side positions. For this experiment, the corresponding task mapping was paired with a mixed mapping, for which the middle positions were assigned to corresponding responses and the side positions to crossed responses (see Figure 5A). Because different responses are required only for the two side positions in the inconsistent conditions of this experiment, its surface structure is more similar to that of the three-choice tasks than is that of the inconsistent corresponding and mirrored mappings used in Experiment 1. However, because the mixed mapping cannot be represented by a single rule, a mapping choice is still needed, even when both tasks use that mapping. If the benefit for consistent mappings arises from eliminating mapping choice, there should be no consistency benefit for the mixed mapping at either side or middle positions. If selection difficulty is a function of the global complexity of the mappings of the eight positions across the two tasks, the inconsistent mapping conditions should yield better performance than will the consistent mixed mapping condition, because only two, rather than four, of the stimulus positions require crossed responses.

\section{Method}

Sixteen new students from the same population and with the same characteristics as those in Experiment 1 participated. These students also participated in Experiment 3 in a different session, 1 week apart, with the order of the experiments counterbalanced. The apparatus, stimuli, and procedure were similar to those in Experiment 1, except for the differences noted. The mixed mapping required participants to press the corresponding key for stimuli at

\section{A Experiment 2: Corresponding and Mixed Mappings}

\section{Consistent Mappings}

Task 1

Task 2

Corresponding-Corresponding

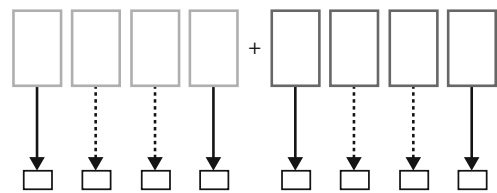

Mixed-Mixed
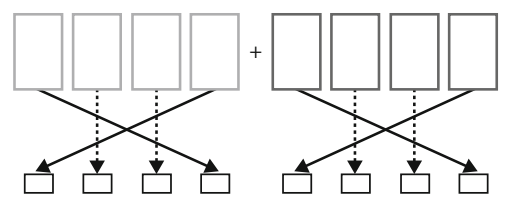

Inconsistent Mappings

Task 1

Task 2

Corresponding-Mixed

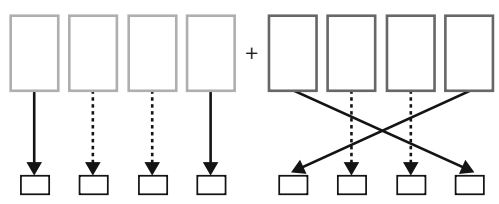

Mixed-Corresponding

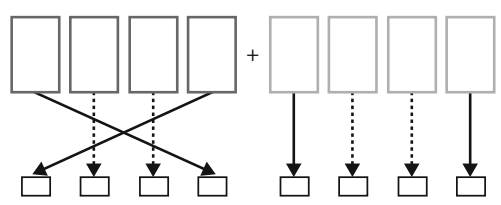

B

Experiment 3: Mirrored and Mixed Mappings

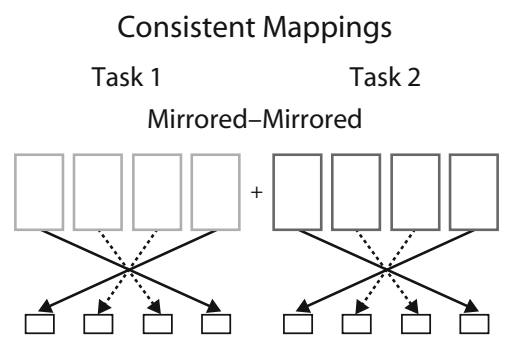

Mixed-Mixed
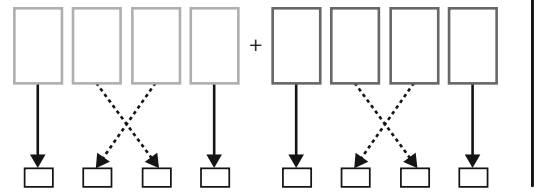

Inconsistent Mappings

Task 1

Task 2

Mirrored-Mixed

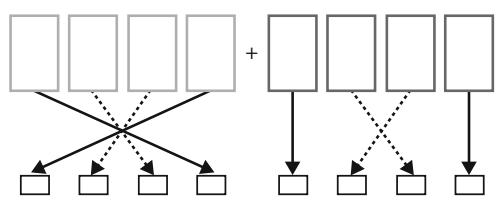

Mixed-Mirrored

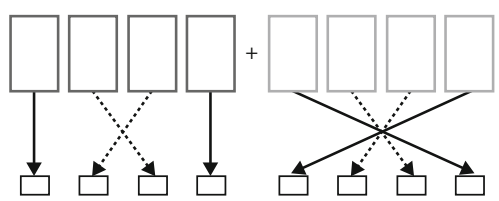

Figure 5. The mappings used in Experiments 2 and 3. (A) The combinations of corresponding and mixed mappings used in Experiment 2. (B) The combinations of mirrored and mixed mappings used in Experiment 3. 
the middle locations and the mirror-opposite key for stimuli at the side locations (see Figure 5A). This mixed mapping was paired with a corresponding mapping for the inconsistent conditions. Thus, the two mappings required the same responses for the middle stimulus positions and different responses for the side stimulus positions. Combinations of corresponding and mixed mappings for Task 1 and Task 2 were performed. The number of test trials within each of the four conditions in each session was 192, as it was in Experiment 1.

\section{Results}

RTs and PEs

Task 1. There were main effects on RT1 of Task 1 mapping $\left[F(1,15)=30.61, M S_{\mathrm{e}}=143,422, p<.001\right]$ and of Task 2 mapping $\left[F(1,15)=10.96, M S_{\mathrm{e}}=173,380, p=\right.$ $.005]$ (see Figures 6A and 6B). For both task mappings, RT1 was shorter when the mapping was corresponding ( $M=928$ and $959 \mathrm{msec}$, respectively) than when it was mixed $(M=1,113$ and $1,081 \mathrm{msec}$, respectively). There was also a position main effect $\left[F(1,15)=17.15, M S_{\mathrm{e}}=\right.$ 29,230, $p<.001]$ : RT1 was shorter for the middle positions $(M=989 \mathrm{msec})$ than for the side positions $(M=$ $1,052 \mathrm{msec})$.
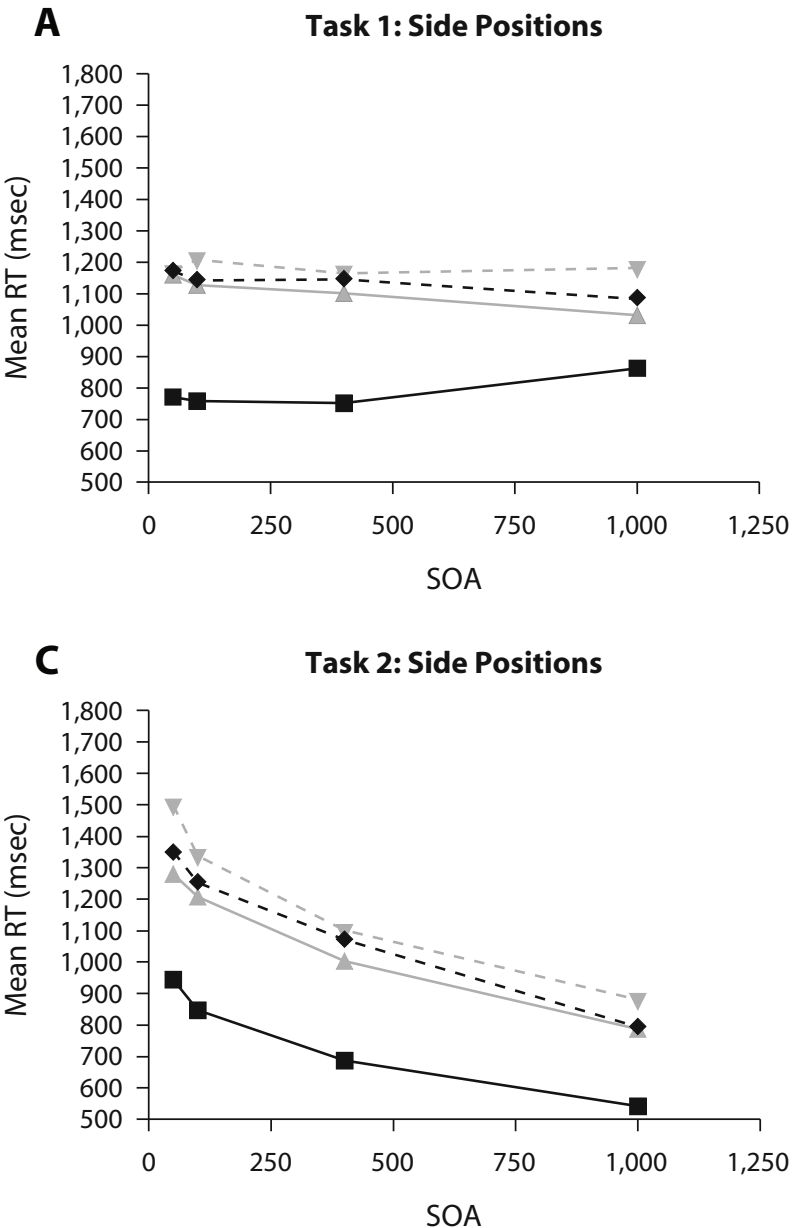

The Task 1 mapping $\times$ Task 2 mapping interaction was not significant $[F(1,15)=2.85, p=.11]$, but the threeway interaction with position was $[F(1,15)=26.05$, $\left.M S_{\mathrm{e}}=47,811, p<.001\right]$. The two mappings interacted for the side positions $[F(1,15)=10.06, p<.006]$, with RT1 being shorter for the corresponding-corresponding condition $(M=786 \mathrm{msec})$ than for the other conditions (mixed-mixed, $M=1,139 \mathrm{msec}$; corresponding-mixed, $M=1,105 \mathrm{msec}$; mixed-corresponding, $M=1,176 \mathrm{msec}$ ) but not for the middle positions $(F<1)$. Analysis of just the corresponding Task 1 mapping showed a significant overall benefit of the Task 2 mappings also being corresponding $[F(1,15)=20.00, p<.001]$, as well as a significant interaction with position, indicative of a larger benefit at the side positions than at the middle positions $[F(1,15)=63.78, p<.001]$.

The main effect of SOA was not significant $[F(3,45)=$ $1.22, p>.30]$, but SOA interacted with Task 2 mapping $\left[F(3,45)=7.54, M S_{\mathrm{e}}=15,794, p<.001\right]$. The benefit for a corresponding Task 2 mapping on RT1 decreased as SOA increased (mean differences $[M D \mathrm{~s}]=152,151,131$, and $32 \mathrm{msec}$, respectively). No other terms were significant.

\section{B}

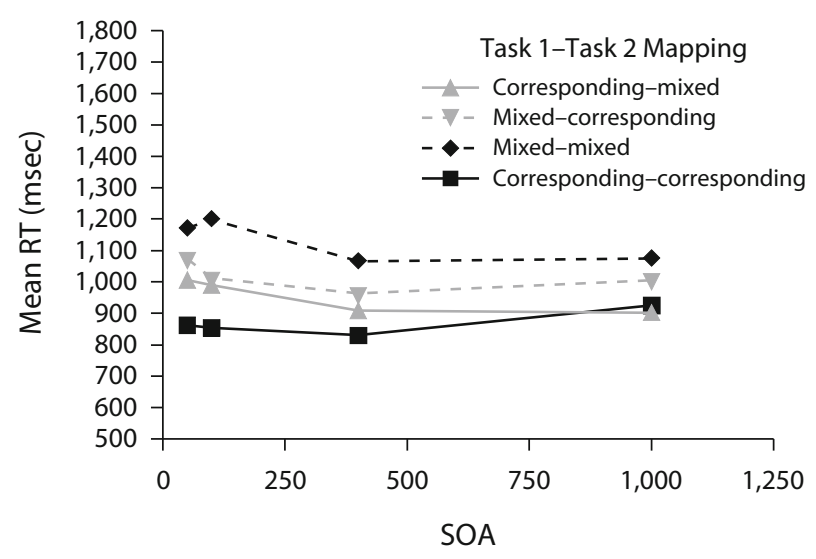

D

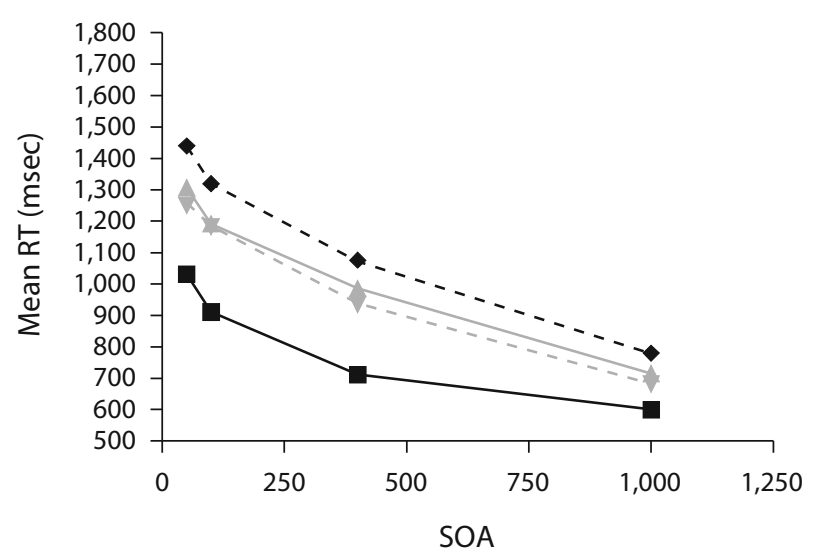

Figure 6. Mean reaction times (RTs) for Task 1 (A and B) and Task 2 (C and D) in milliseconds for the side (A and C) and middle (B and D) positions as a function of Task 1-Task 2 mapping and stimulus onset asynchrony (SOA) for the corresponding and mixed mappings in Experiment 2. 
Table 2

Mean Percentages of Error for Task 1 and Task 2 As a Function of Task 1-Task 2 Mapping and Stimulus Onset Asynchrony (SOA, in Milliseconds) for the Side Positions of Experiments 2 and 3

\begin{tabular}{lcccc}
\hline & \multicolumn{4}{c}{ SOA } \\
\cline { 2 - 5 } Task 1-Task 2 Mapping & 50 & 150 & 400 & 1,000 \\
\hline & Experiment 2 & & \\
Task 1 & & & & \\
Corresponding-corresponding & 1.04 & 1.04 & 1.04 & 1.57 \\
Corresponding-mixed & 4.73 & 4.69 & 5.99 & 3.91 \\
Mixed-corresponding & 4.98 & 6.52 & 4.95 & 5.49 \\
Mixed-mixed & 1.04 & 0.78 & 2.34 & 3.40 \\
Task 2 & & & & \\
Corresponding-corresponding & 2.87 & 2.34 & 2.34 & 1.04 \\
Corresponding-mixed & 7.81 & 4.74 & 6.51 & 7.29 \\
Mixed-corresponding & 8.94 & 7.13 & 5.36 & 7.03 \\
Mixed-mixed & 3.14 & 6.61 & 4.17 & 3.65 \\
& & & & \\
Task 1 & Experiment 3 & & & \\
Mirrored-mirrored & & & & \\
Mirrored-mixed & 2.63 & 1.57 & 2.89 & 2.08 \\
Mixed-mirrored & 4.06 & 3.16 & 1.34 & 2.40 \\
Mixed-mixed & 6.08 & 9.52 & 9.41 & 10.5 \\
Task 2 & 2.34 & 1.60 & 2.36 & 3.15 \\
Mirrored-mirrored & & & & \\
Mirrored-mixed & 3.39 & 3.79 & 4.77 & 1.57 \\
Mixed-mirrored & 8.92 & 8.57 & $11.6 *$ & 11.1 \\
Mixed-mixed & 5.92 & 6.08 & 7.00 & 8.20 \\
\hline & 1.85 & 1.56 & 2.91 & 2.08 \\
\hline
\end{tabular}

For PE1 (see Table 2), the only significant effects were the Task 1 mapping $\times$ Task 2 mapping interaction $\left[F(1,15)=5.75, M S_{\mathrm{e}}=43.04, p=.030\right]$ and the threeway interaction of those variables with position $[F(1,15)=$ $\left.9.21, M S_{\mathrm{e}}=69.38, p=.008\right]$. The task mappings interacted for both side and middle positions $\left[F_{\mathrm{s}}(1,15)=7.91\right.$ and $7.50, p \mathrm{~s}=.013$ and .015 , respectively]. For the side positions, PE1 was lower when the task mappings were consistent $(M=1.54 \%)$ than when they were inconsistent $(M=5.16 \%)$. For the middle positions, the differences in PE1 across conditions were smaller, and PE1 was higher for the mixed-mixed condition $(M=2.88 \%)$ than for the other three conditions $(M=1.53 \%)$.

Task 2. RT2 showed main effects of Task 1 mapping $\left[F(1,15)=81.06, M S_{\mathrm{e}}=62,350, p<.001\right]$ and of Task 2 mapping $\left[F(1,15)=25.87, M S_{\mathrm{e}}=115,873, p<.001\right]$ (see Figures $6 \mathrm{C}$ and $6 \mathrm{D}$ ), but these were modified by a Task 1 mapping $\times$ Task 2 mapping interaction $[F(1,15)=17.89$, $\left.M S_{\mathrm{e}}=106,243, p<.001\right] . \mathrm{RT} 2$ was shorter when the mappings were both corresponding $(M=785 \mathrm{msec})$ than when they were not $(M \mathrm{~s}=1,059,1,105$, and $1,136 \mathrm{msec}$ for the corresponding-mixed, mixed-corresponding, and mixed-mixed conditions, respectively).

The main effect of position approached the .05 level $\left[F(1,15)=4.21, M S_{\mathrm{e}}=23,806, p=.058\right]$, and position entered into two- and three-way interactions with Task 1 mapping and Task 2 mapping $\left[F_{\mathrm{s}}(1,15)>6.00\right.$, $p$ s $<.027]$. The two-way interaction of Task 1 mapping and Task 2 mapping was significant for the side positions $[F(1,15)=26.13, p<.001]$ but not for the middle positions $[F(1,15)=3.32, p=.089]$, although the corresponding mapping still showed a $198-\mathrm{msec}$ benefit of the
Task 1 mapping's also being corresponding at the middle positions.

There was a main effect of SOA $[F(3,45)=299.98$, $\left.M S_{\mathrm{e}}=24,362, p<.001\right]$, showing a PRP effect for which RT2 was longer at short SOAs than at long ones $(M \mathrm{~s}=$ $1,252,1,156,946$, and $722 \mathrm{msec}$, respectively). SOA entered into two-way and three-way interactions with Task 1 mapping and Task 2 mapping $[F \mathrm{~s}(3,45)>3.41$, $p \mathrm{~s}<.025]$ (see Figures 6C and 6D). As in Experiment 1, RT2 decreased least across SOA for the correspondingcorresponding condition, which had the shortest RTs. A separate ANOVA performed on the other three conditions showed no significant difference between those conditions $[F(2,30)=1.93, p>.15]$ and no interaction with SOA $[F(6,90)=1.44, p>.20]$.

For PE2, the only main effect was that of $\operatorname{SOA}[F(3,45)=$ 2.96, $\left.M S_{\mathrm{e}}=18.14, p=.042\right]$. PE2 showed a PRP effect, with PE2 decreasing as SOA increased $(5.87 \%, 4.69 \%$, $4.40 \%$, and $3.78 \%$, respectively). Also, Task 1 mapping interacted with Task 2 mapping $\left[F(1,15)=9.37, M S_{\mathrm{e}}=\right.$ $31.12, p=.008]$, and these variables entered into a threeway interaction with position $\left[F(1,15)=9.35, M S_{\mathrm{e}}=\right.$ $58.90, p=.008]$ and a four-way interaction with position and SOA $\left[F(3,45)=3.16, M S_{\mathrm{e}}=20.28, p=.035\right]$. PE2 showed a consistency benefit for the side positions $[F(1,15)=12.61, p=.003]$ but not for the middle ones $(F<1)$. For the side positions, PE2 was lower when the task mappings were consistent $(M=3.27 \%)$ than when they were not $(M=6.85 \%)$. The consistency benefit for the side positions was mainly evident at the longest SOA (see Table 2) $[F(3,45)=2.96, p=.042]$; the lack of a consistency benefit for middle positions did not vary across $\operatorname{SOA}[F(3,45)=21.48, p>.20]$.

\section{R-R Compatibility}

$\mathrm{R}-\mathrm{R}$ compatibility was analyzed similarly to that in Experiment 1 (see Appendix A). For RT1 (see Figures 7A and 7B), the most important significant terms involving $\mathrm{R}-\mathrm{R}$ compatibility were its three-way interaction with Task 1 mapping and middle versus side position and the four-way interaction with those factors and Task 2 mapping. An R-R compatibility effect was evident only for the combination of mixed mapping and middle position: RT1 was similar for the compatible and mirror-opposite relations and longer for the incompatible relation. This pattern manifested primarily when the Task 2 mapping was corresponding.

For RT2 (see Figures 7C and 7D), several terms involving $\mathrm{R}-\mathrm{R}$ compatibility were significant, including the four-way interaction of all variables. For the consistent corresponding condition and the two inconsistent mapping conditions, RT2 was shorter when R1 position was compatible or mirror-opposite than when it was incompatible. This difference was evident for the side positions in the consistent corresponding condition and for the middle positions in the inconsistent conditions. Finally, for the consistent mixed condition, R-R compatibility effects were evident at both middle and side positions, with the mirror-opposite relation yielding shorter RT2 than the incompatible and compatible relations. 


\section{A}

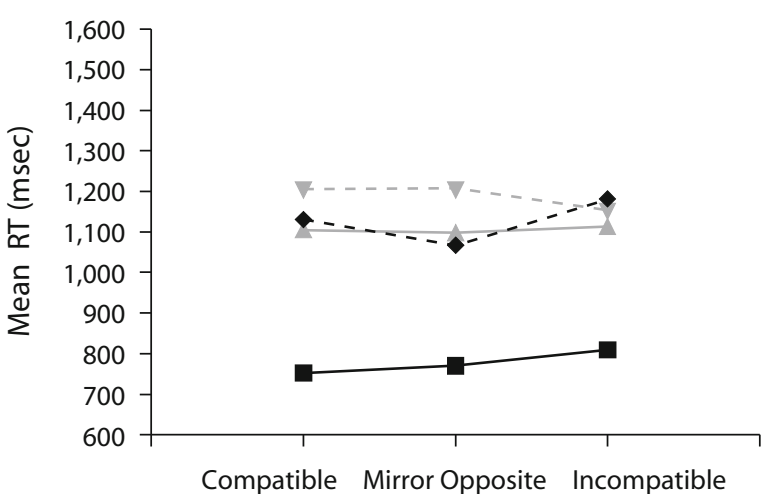

R-R Compatibility Relation

C

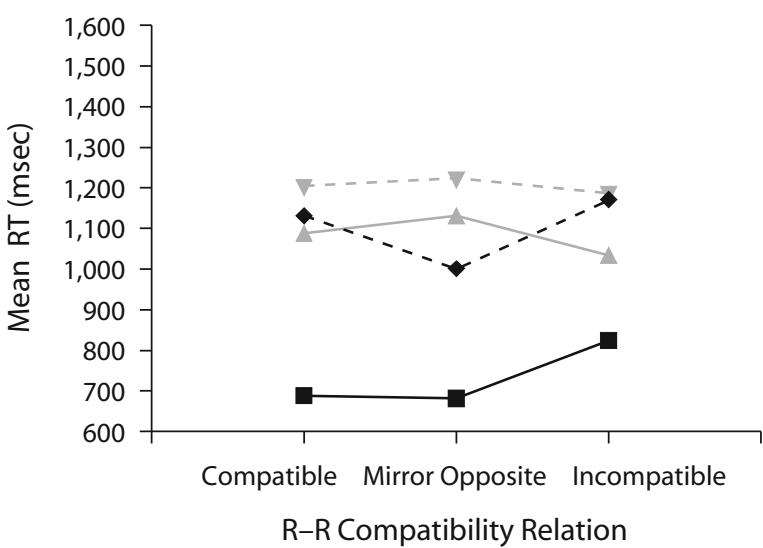

B

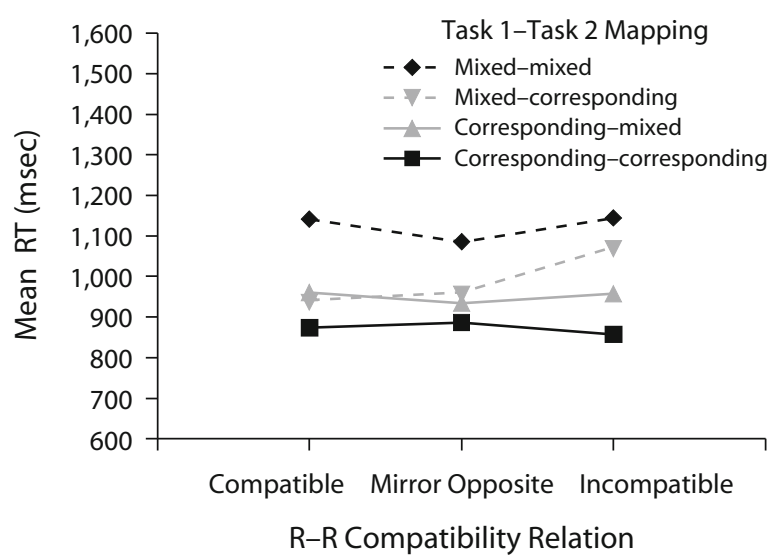

D

Task 2: Middle Positions

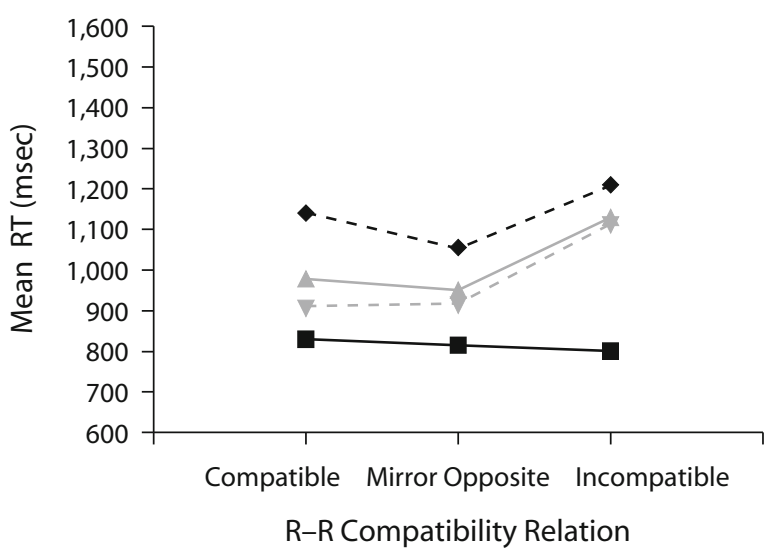

Figure 7. Mean reaction times (RTs) for Task 1 (A and B) and Task 2 (C and D) in milliseconds for the side (A and C) and middle (B and D) stimulus positions as a function of response-response (R-R) compatibility and Task 1-Task 2 mappings in Experiment 2.

To summarize, as in Experiment 1, there was little sign of R-R compatibility effects for individual response locations. Rather, the benefits were for correspondence of middle versus side responses, suggesting that a middle-orside decision is part of the response-selection process.

\section{Error Types}

For the consistent mapping conditions and the two middle positions in the inconsistent conditions, an incorrect response can be in the mirror-opposite position, an adjacent position (nonmirror opposite for middle responses), or another position that is neither adjacent nor mirror opposite. For the inconsistent mapping side positions, the mirror-opposite response is also the one that would be correct for the alternative mapping rule. The frequencies of the incorrect response types are shown in Figure 8 (see Appendix B for the full analysis).

The most important feature of the data can be seen by comparing the side positions (Figures $8 \mathrm{~A}$ and $8 \mathrm{C}$ ) with the middle positions (Figures $8 \mathrm{~B}$ and $8 \mathrm{D}$ ). At the side positions, for which the responses for the two mappings differed, many errors in the inconsistent conditions involved making the response that was correct for the alternative mapping. Although at the middle positions, for which the correct response was corresponding for both mappings, the frequency of responding with the noncorresponding middle location was only slightly greater than the frequency of responding with the adjacent side response. For consistent mixed mappings, the most frequent errors were to make the corresponding response to stimuli in the side positions and with the crossed middle response to stimuli in the middle positions. For consistent corresponding mappings, most errors involved pressing an adjacent key. Thus, the error analysis shows that, with the mixed mapping, there is a cost associated with an emergent choice between mapping rules within the task but not between a task using the mixed mapping and one using a corresponding mapping.

\section{Discussion}

For the side positions, at which the responses for the corresponding and mixed mappings differed, performance on Tasks 1 and 2 was better when both mappings were corresponding than when at least one mapping was not. Neither RT1 nor RT2 showed a benefit of maintaining 
A

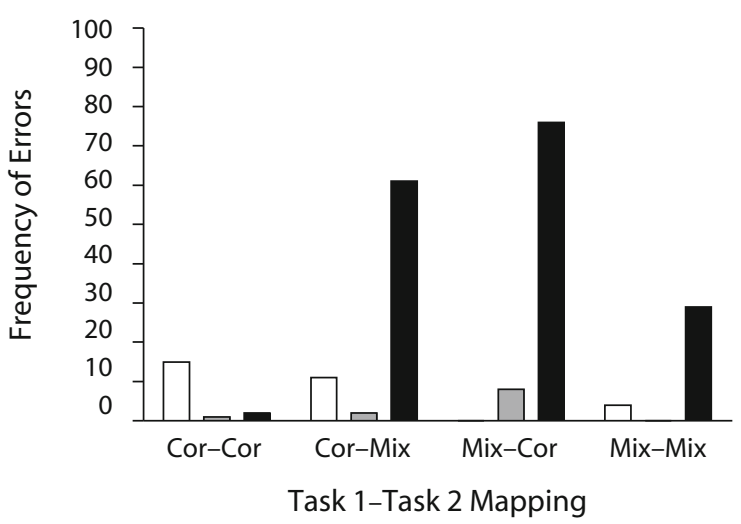

C

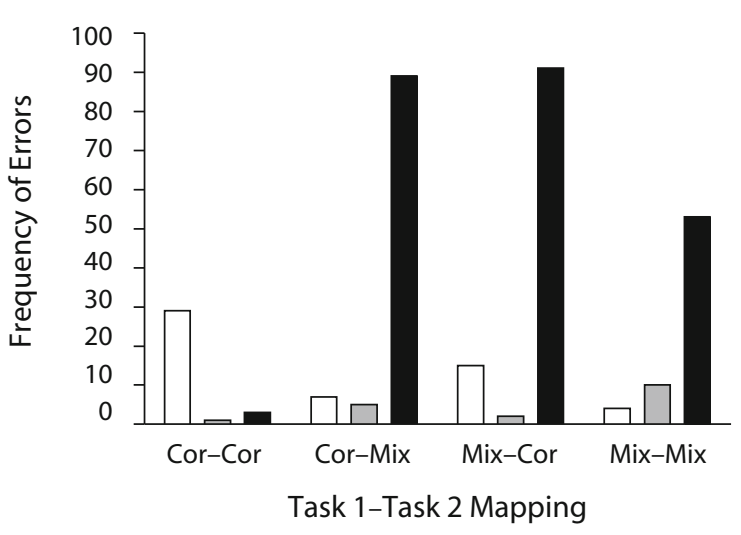

B

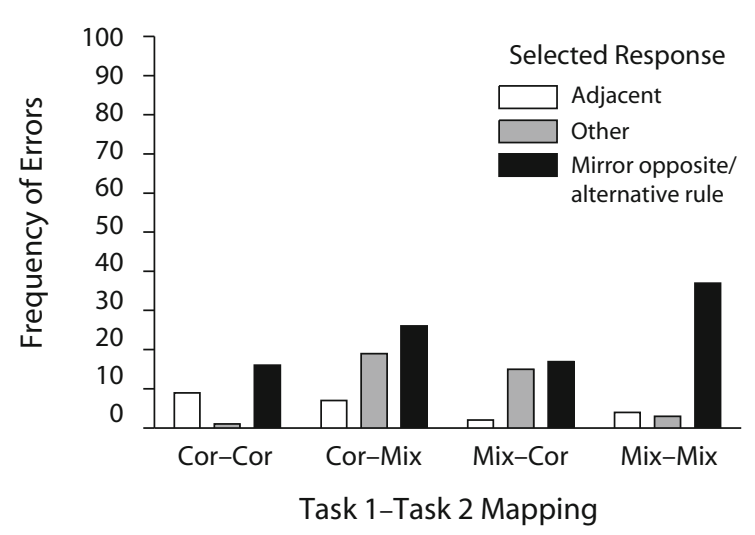

D

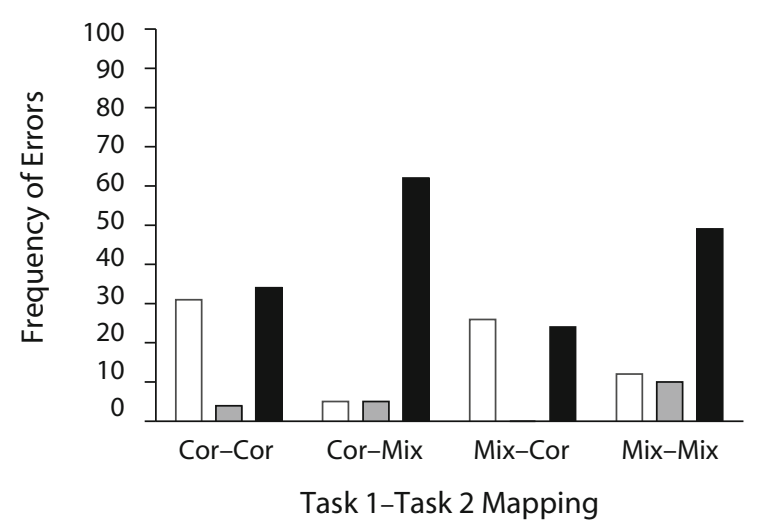

Figure 8. Frequencies of errors for Task 1 (A and B) and Task 2 (C and D) as a function of Task 1-Task 2 mapping and selected response in Experiment 2. Cor, corresponding mapping; Mix, mixed mapping.

consistent mixed mappings for the two tasks, which is in agreement with the emergent mapping-choice account if it is assumed that each task must conform to the same mapping rule in order to bypass rule selection. However, PEs were higher for the inconsistent conditions in which the mixed mapping was paired with the corresponding mapping than for the mixed-mixed condition. Thus, although the RT data showed no consistency benefit for the mixed mapping, the PE data did.

For the middle positions, for which the same responses were required regardless of whether the task mapping was corresponding or mixed, performance was better when the Task 1 or Task 2 mapping was corresponding than when it was not, but there was no interaction indicative of an overall consistency benefit. There was again a benefit from both tasks using a corresponding mapping beyond that which could be attributed to the separate advantages of correspondence for each individual task. Moreover, there was no benefit for maintaining a consistent mixed mapping; this condition yielded the longest RTs and highest PEs. Thus, the lower PE for the mixed-mixed condition than for the inconsistent conditions evident for the side positions was not apparent for the middle positions.

The absence of a consistency benefit in RT1 or RT2 for the mixed-mixed condition at the middle stimulus posi- tions is consistent with the emergent mapping-choice account, as is the absence for the side positions, because rule selection is still needed when both tasks use the mixed mapping. Although the RT data did not show a consistency benefit with the mixed mapping at the side positions, the PE data did. This outcome of a PE consistency benefit at the side positions but not at the middle ones is similar to the pattern that we observed for three-choice tasks, in which the mirror-opposite mapping showed a consistency benefit at the side positions but not at the middle position, for which the response was the same as that with the corresponding mapping (Proctor \& Vu, 2008). Thus, the PE data suggest that, when mappings are mixed, an initial choice is made between middle or side positions, and if the decision is "side," the corresponding or opposite mapping rule is selected and applied.

\section{EXPERIMENT 3}

In Experiment 3, the task mapping was mirrored for all four locations or mixed, with the middle positions assigned to crossed responses and the side positions to corresponding responses (see Figure 5B). These tasks also require the same responses for the two middle positions, but, in this case, the responses are crossed. A consistency 
benefit should be apparent for both task mappings at the side positions but not at the middle positions if the consistency benefit is due to having different responses for the specific pair of positions across the two task mappings. The emergent mapping-choice account predicts a consistency benefit for the mirrored-mirrored condition, because it can be characterized by a single mapping rule. No such benefit for the mixed-mixed condition should be found if the individual task mappings must be characterized by a single rule. However, a benefit would be found if complexity of the global mapping of the eight stimulus positions to responses is the critical factor.

All previous demonstrations of consistency benefits for a mirrored mapping have been obtained relative to conditions in which that mapping was paired with a pure corresponding mapping. The combination of mirrored and mixed mappings for the two tasks thus allows determination of whether a benefit can be obtained for consistent mirrored mappings relative to inconsistent conditions, for which the mirrored mapping is paired with one for which only some $\mathrm{S}-\mathrm{R}$ assignments are crossed.

Studies in which corresponding and mirrored mappings are mixed within a single task show a mixing cost on RTs that is greater for corresponding trials than for opposite trials (Shaffer, 1965; Vu \& Proctor, 2004; Yamaguchi \& Proctor, 2006). Thus, it is possible that the mapping consistency benefit for dual tasks is mainly a consequence of mixing a corresponding mapping with one that is not; in which case, the benefit should not be evident in Experiment 3.

\section{Method}

As previously stated, the same 16 participants from Experiment 2 engaged in Experiment 3. For the mixed mapping in this experiment, they were required to press the mirror-opposite key for stimuli at the middle locations and the corresponding key for stimuli at the side locations. This was paired with a mirrored mapping for the inconsistent conditions. Thus, as in Experiment 2, the two mappings required the same responses for the middle positions and different responses for the side positions. Combinations of mirrored and mixed mappings were performed, with the number of test trials within each of the four conditions being 192, as it was in Experiment 2.

\section{Results}

\section{RTs and PEs}

Task 1. The Task 1 mapping $\times$ Task 2 mapping interaction for RT1 was significant $\left[F(1,15)=37.41, M S_{\mathrm{e}}=\right.$ $130,563, p<.001]$, as was the three-way interaction with position $\left[F(1,15)=21.72, M S_{\mathrm{e}}=63,658, p<.001\right]$. Both side and middle positions showed consistency benefits $\left[F(1,15)=48.07\right.$ and 7.13, respectively; $M S_{e} \mathrm{~s}=119,251$ and 74,970, respectively; $p$ s $<.001$ and .017 , respectively], with the benefits being larger for the side positions $(M \mathrm{~s}=$ 1,049 vs. $1,348 \mathrm{msec}$; see Figure $9 \mathrm{~A}$ ) than for the middle positions ( $M \mathrm{~s}=1,109$ vs. 1,200 msec; see Figure 9B).

The three-way interaction of position, Task 1 mapping, and SOA was significant $\left[F(3,45)=3.08, M S_{\mathrm{e}}=14,151\right.$, $p=.037$, but not with the Huynh-Feldt correction ( $p=$ .056 ). For the side positions, the mirrored Task 1 mapping tended to have longer RTs than did the mixed Task 1 map-
Table 3

Mean Percentages of Error for Task 1 and Task 2 As a Function of Task 1-Task 2 Mapping and Stimulus Onset Asynchrony (SOA, in Milliseconds) for the Middle Positions of Experiments 2 and 3

\begin{tabular}{lllll} 
& \multicolumn{4}{c}{ SOA } \\
\cline { 2 - 5 } Task 1-Task 2 Mapping & 50 & 150 & 400 & 1,000
\end{tabular}

Experiment 2

\begin{tabular}{|c|c|c|c|c|}
\hline Corresponding-corresponding & 1.85 & 2.34 & 1.30 & 1.30 \\
\hline Corresponding-mixed & 1.63 & 2.08 & 1.31 & 1.82 \\
\hline Mixed-corresponding & 1.30 & 0.78 & 1.86 & 0.78 \\
\hline Mixed-mixed & 2.60 & 3.20 & 3.13 & 2.60 \\
\hline \multicolumn{5}{|l|}{ Task 2} \\
\hline Corresponding-corresponding & 8.14 & 4.23 & 2.60 & 2.90 \\
\hline Corresponding-mixed & 5.38 & 5.36 & 5.01 & 3.39 \\
\hline Mixed-corresponding & 5.21 & 2.63 & 4.48 & 0.78 \\
\hline Mixed-mixed & 5.49 & 4.29 & 4.71 & 4.18 \\
\hline \multicolumn{5}{|c|}{ Experiment 3} \\
\hline \multicolumn{5}{|l|}{ Task 1} \\
\hline Mirrored-mirrored & 1.57 & 0.00 & 0.78 & 1.04 \\
\hline Mirrored-mixed & 0.26 & 1.35 & 0.52 & 0.55 \\
\hline Mixed-mirrored & 2.01 & 1.86 & 0.00 & 0.78 \\
\hline Mixed-mixed & 2.34 & 1.04 & 1.56 & 3.17 \\
\hline \multicolumn{5}{|l|}{ Task 2} \\
\hline Mirrored-mirrored & 2.11 & 2.65 & 3.42 & 0.52 \\
\hline Mirrored-mixed & 3.96 & 4.51 & 5.66 & 3.42 \\
\hline Mixed-mirrored & 3.02 & 2.94 & 2.13 & 2.65 \\
\hline Mixed-mixed & 6.04 & 4.98 & 4.44 & 2.34 \\
\hline
\end{tabular}

ping, but this difference largely disappeared at the longest SOA.

For PE1 (see Table 3), main effects of position $[F(1,15)=$ 5.60, $\left.M S_{e} \mathrm{~s}=191, p=.032\right]$ and Task 2 mapping $[F(1,15)=$ $\left.7.10, M S_{e} \mathrm{~s}=33, p=.018\right]$ were significant. PE1 was higher for the side positions $(M=4.07 \%)$ than for the middle positions $(M=1.18 \%)$ and for the mirrored mapping $(M=3.29 \%)$ than for the mixed mapping $(M=1.95 \%)$. The Task 1 mapping $\times$ Task 2 mapping interaction was significant $\left[F(1,15)=4.87, M S_{\mathrm{e}}=58, p=.043\right]$, as was the three-way interaction with position $\left[F(1,15)=5.62, M S_{\mathrm{e}}=\right.$ $91, p=.032]$. For the side positions, PE1 was lower when the task mappings were consistent $(M=2.32 \%)$ than when they were not $(M=5.81 \%)[F(1,15)=5.47, p=.034]$, but this difference was not significant for the middle positions $[F(1,15)=2.46, p>.13]$.

The final significant interaction for PE1 was that of position, Task 2 mapping, and SOA $\left[F(3,45)=3.76, M S_{\mathrm{e}}=\right.$ $7.80, p=.017]$. For the side positions, more errors were made at the longest SOA than at the shortest SOA when the Task 2 mapping was mirrored $(M D=1.93 \%)$ than when it was mixed ( $M D=0.42 \%)$; for the middle positions, more errors were made at the shortest SOA than at the longest SOA when the mapping was mirrored $(M D=$ $0.88 \%$ ), but more errors were made at the longest SOA when the mapping was mixed $(M D=0.55 \%)$

Task 2. Position showed a main effect $[F(1,15)=8.89$, $\left.M S_{\mathrm{e}}=40,617, p<001\right]:$ RT2 was shorter for the middle positions $(M=1,170 \mathrm{msec})$ than for the side positions ( $M=$ $1,223 \mathrm{msec})$. Position also interacted with Task 1 mapping $\left[F(1,15)=6.25, M S_{\mathrm{e}}=10,746, p=025\right]: \mathrm{RT}$ was $79 \mathrm{msec}$ shorter for the mixed mapping than for the mirrored map- 

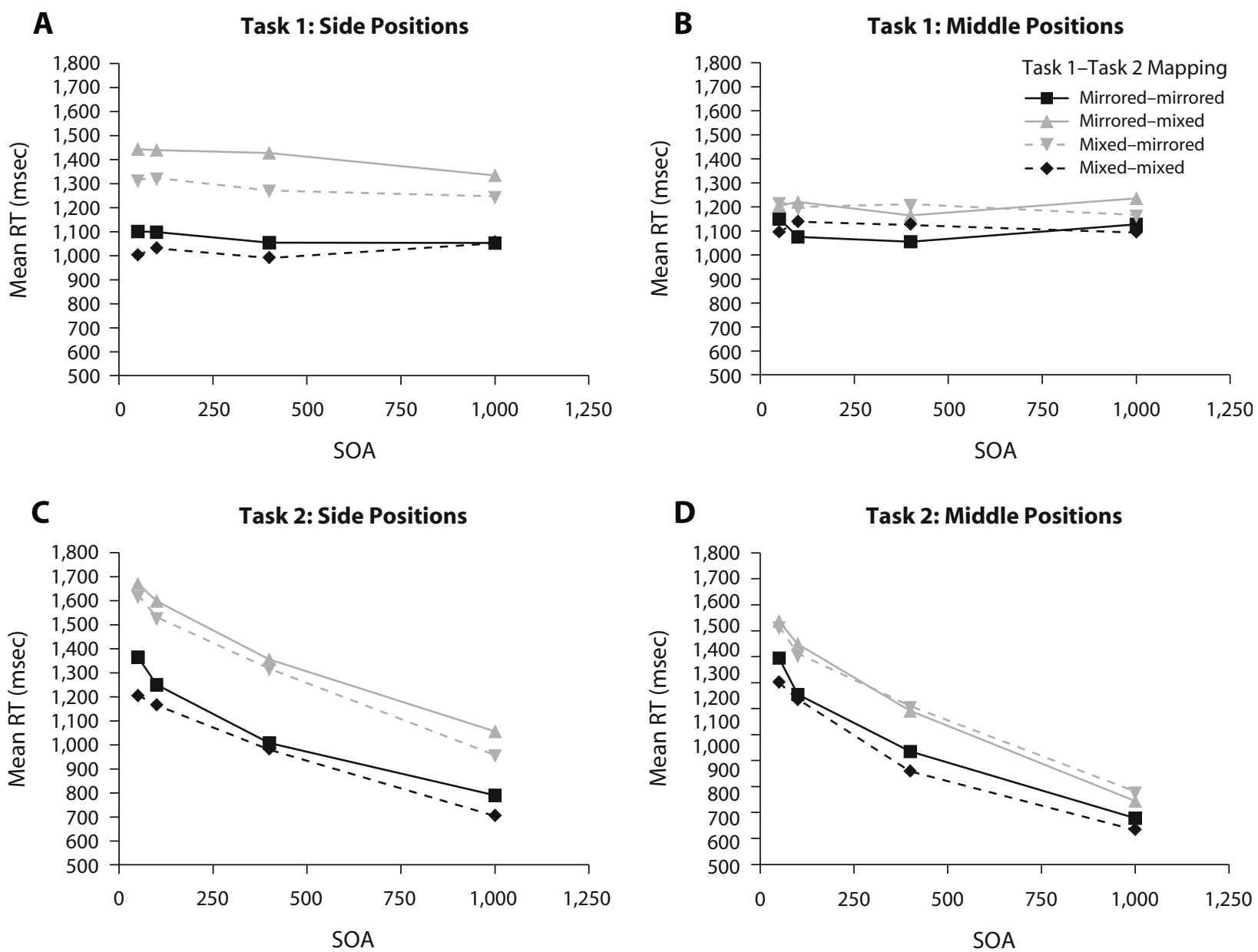

Figure 9. Mean reaction times (RTs) in Task 1 (A and B) and Task 2 (C and D) in milliseconds for side (A and C) and middle (B and D) positions as a function of Task 1-Task 2 mapping and stimulus onset asynchrony (SOA) for the mirrored and mixed mappings in Experiment 3.

ping at the side positions but was only $33 \mathrm{msec}$ shorter at the middle positions.

Task 1 mapping interacted with Task 2 mapping $\left[F(1,15)=33.02, M S_{\mathrm{e}}=233,626, p<.001\right]$, but this was qualified by a three-way interaction $[F(1,15)=34.58$, $\left.M S_{\mathrm{e}}=24,410, p<.001\right]$ (see Figure 9C and 9D). Similar to what was found for RT1, a consistency benefit was apparent for both the side $(M D=327 \mathrm{msec})$ and middle $(M D=165 \mathrm{msec})$ positions $[F \mathrm{~s}(1,15)=73.03$ and 10.50, respectively; $M S_{e} \mathrm{~s}=93,539$ and 164,497 , respectively; $p$ s $<.005]$, but the effect was smaller for the middle positions.

The main effect of SOA was also significant $[F(3,45)=$ $\left.422.39, M S_{\mathrm{e}}=22,306, p<.001\right]$, showing a PRP effect: Overall, RT2 was longer at short SOAs than at long ones $(M \mathrm{~s}=1,450,1,360,1,131$, and $842 \mathrm{msec}$, respectively). SOA interacted with Task 1 and Task 2 mapping $\left[F(3,45)=4.17, M S_{\mathrm{e}}=9,557, p=.011\right]$. The PRP effect was larger for the inconsistent mappings $(601 \mathrm{msec})$ than for the consistent mappings (565 $\mathrm{msec})$.

For PE2 (see Table 3$)$, the effects of position $[F(1,15)=$ $\left.5.36, M S_{\mathrm{e}}=111, p=.035\right]$ and of SOA $[F(3,45)=3.05$,
$\left.M S_{\mathrm{e}}=12, p=.038\right]$ were significant, as was their interaction $\left[F(3,45)=4.81, M S_{\mathrm{e}}=8, p=.005\right]$. Fewer errors were made for the middle positions $(M=3.42 \%)$ than for the side positions $(M=5.58 \%)$, and PE2 was $4.40 \%$, $4.39 \%, 5.24 \%$, and $3.98 \%$ for SOAs of $50,150,400$, and $1,000 \mathrm{msec}$, respectively. SOA had significant effects for both the side $\left[F(3,45)=3.22, M S_{\mathrm{e}}=11, p=.031\right]$ and middle $\left[F(3,45)=4.45, M S_{\mathrm{e}}=9, p=.008\right]$ positions, but with different patterns. For the side positions, PE2 tended to be lower at the two shortest SOAs than at the two longest ones $(M \mathrm{~s}=5.01 \%, 5.00 \%, 6.57 \%$, and $5.73 \%$ for SOAs of 50, 150, 400, and 1,000 msec, respectively), whereas, for the middle positions, PE2 was higher at the three shortest SOAs than at the longest one $(M \mathrm{~s}=3.78 \%$, $3.77 \%, 3.91 \%$, and $2.23 \%$, respectively).

Task 1 mapping interacted with Task 2 mapping $\left[F(1,15)=8.05, M S_{\mathrm{e}}=138, p=.012\right]$, and these variables entered into a three-way interaction with position $\left[F(1,15)=7.17, M S_{\mathrm{e}}=133, p=.017\right]$. A consistency benefit of $5.68 \%$ was evident for the side positions $[F(1,15)=$ $8.67, p=.010]$, but there was no consistency benefit for the middle positions $(F<1)$. 


\section{R-R Compatibility}

Analysis of R-R compatibility for RT1 (see Appen$\operatorname{dix} \mathrm{A}$ ) showed a three-way interaction of R-R compatibility with Task 1 mapping and Task 2 mapping and a four-way interaction of those variables with position. R-R compatibility effects were evident for the consistent mapping conditions but not for the inconsistent ones (see Figures $10 \mathrm{~A}$ and $10 \mathrm{~B}$ ). For both the consistent mirrored and the consistent mixed conditions, RT2 was shorter for the mirror-opposite relation than for the incompatible relation, with the compatible relation being intermediate and not significantly different from either. The four-way interaction is due to the pattern for the consistent mirrored condition holding most strongly for the middle positions and to that for the consistent mixed condition holding mainly for the side positions.

For RT2 (see Figure 10C and 10D), there were threeway $\mathrm{R}-\mathrm{R}$ compatibility $\times$ Task 1 mapping $\times$ Task 2 mapping and R-R compatibility $\times$ Task 1 mapping $\times$ position interactions, as well as a four-way interaction of all variables. For the consistent mirrored mappings, R-R compatibility effects were evident for middle and side positions:

\section{A}

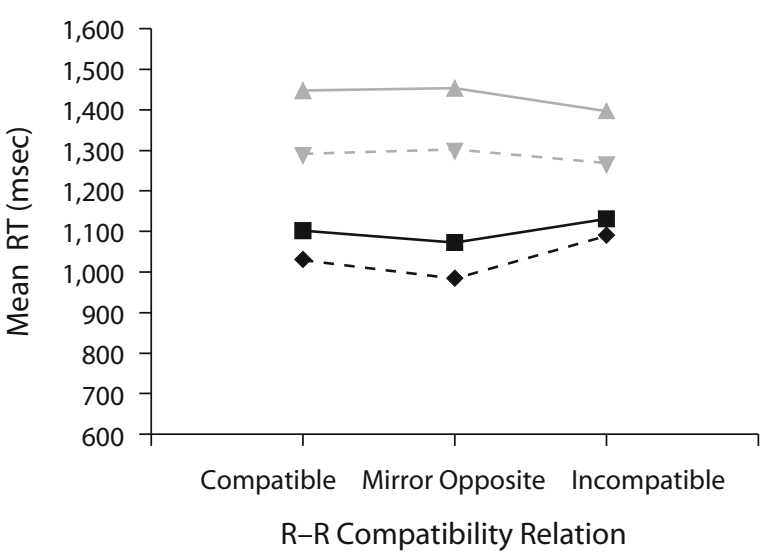

C

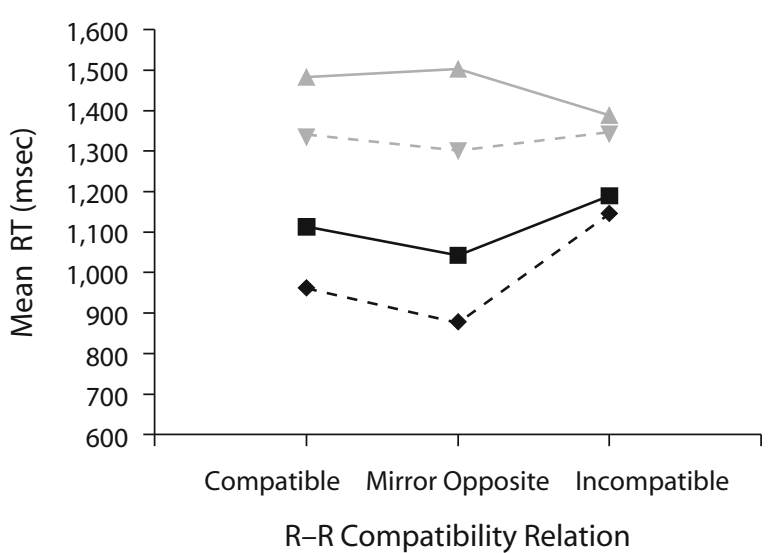

For both positions, the only significant difference was between mirror-opposite and incompatible conditions, with the compatible condition being intermediate. For the inconsistent mappings, R-R compatibility effects were evident only for the middle positions: RT2 did not differ significantly between when R1 position was mirror-opposite and when it was compatible, but both were shorter than when it was incompatible. For the consistent mixed condition, R-R compatibility effects were evident at the side positions, where compatible and mirror-opposite relations yielded a shorter RT2 than did the incompatible condition (see Figure 10C and 10D).

\section{Error Types}

For the consistent mapping conditions, errors tended to be predominantly the mirror-opposite response (see Figure 11 and Appendix B). For the inconsistent mapping conditions, the frequency of those errors increased, particularly for the mixed mapping side responses, regardless of whether the mixed mapping was for Task 1 or Task 2. For the side positions, the correct response for the mixed mapping is corresponding, which means that participants

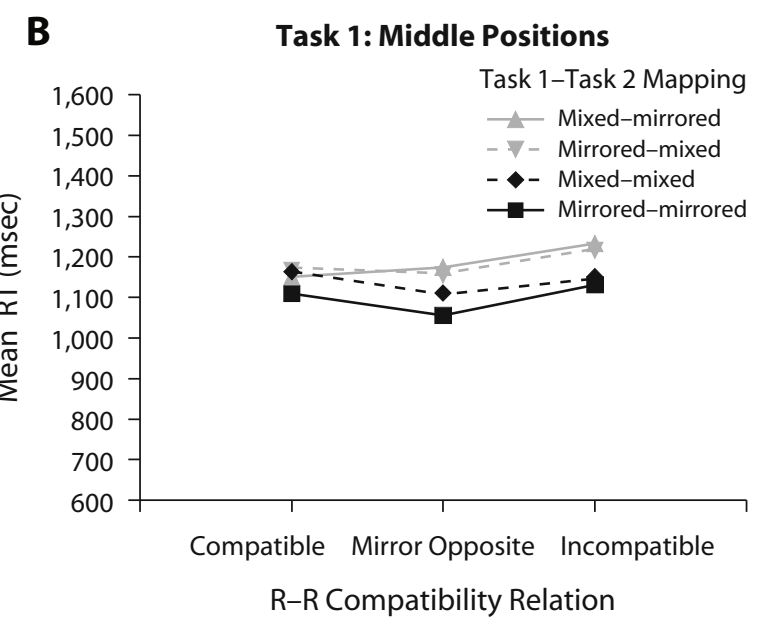

D

Task 2: Middle Positions

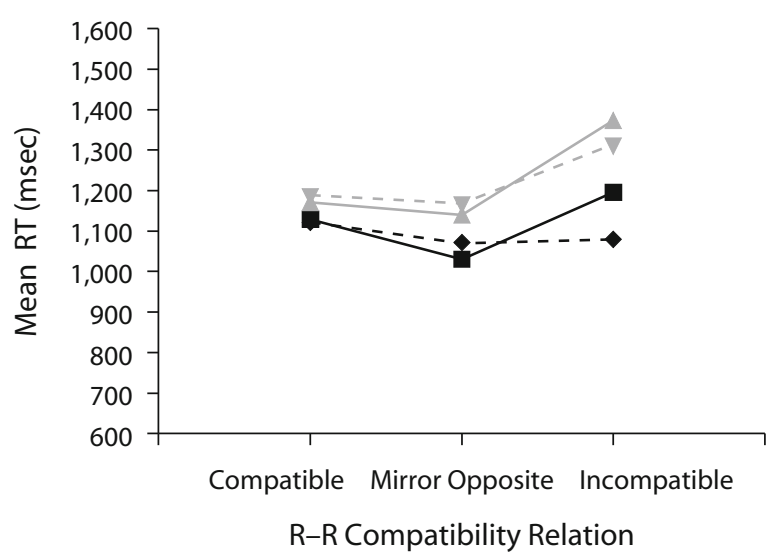

Figure 10. Mean reaction times (RTs) in Task 1 (A and B) and Task 2 (C and D) in milliseconds for the side (A and C) and middle (B and D) stimulus positions as a function of response-response $(R-R)$ compatibility and Task 1-Task 2 mappings in Experiment 3. 

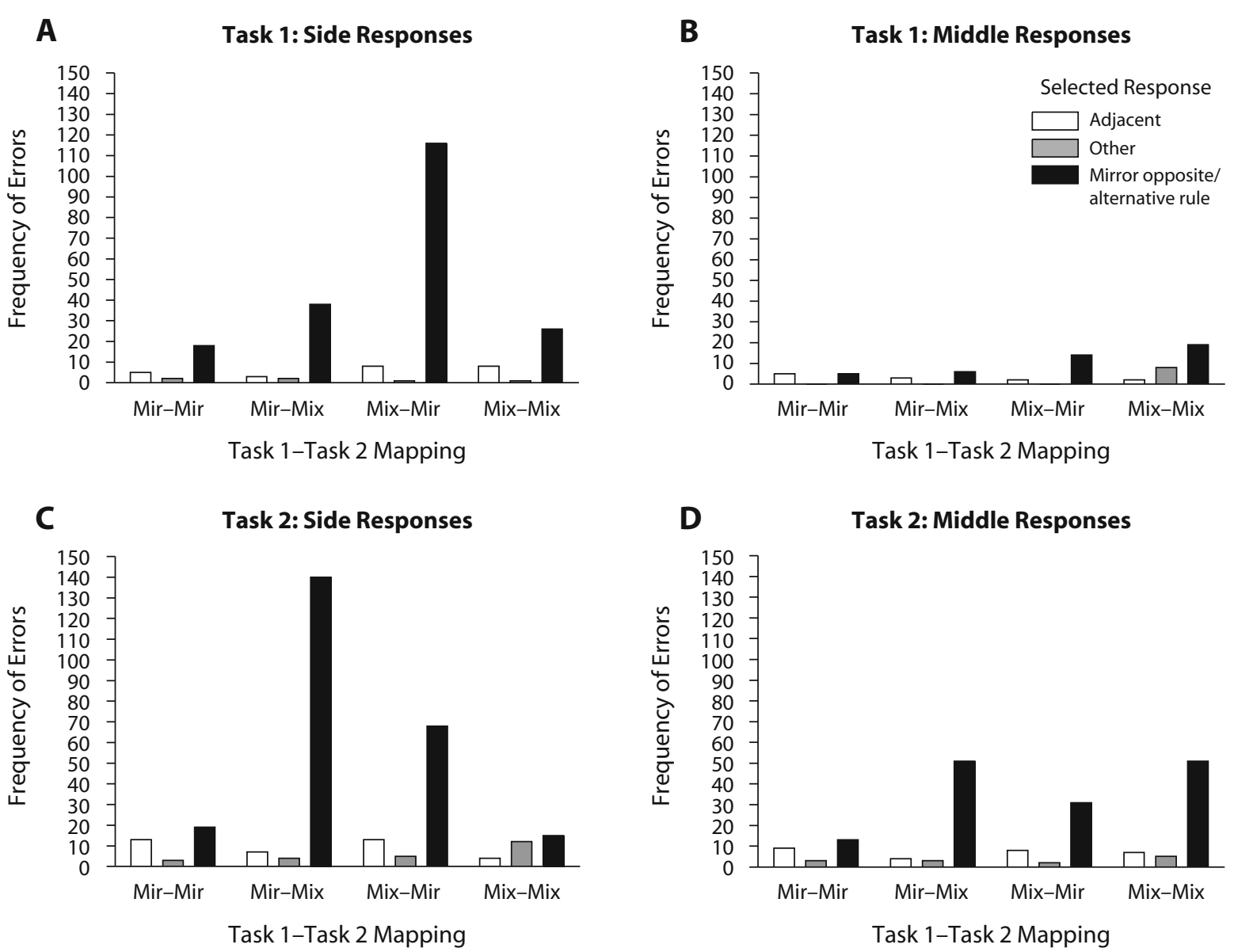

Figure 11. Frequencies of errors for Task 1 (A and B) and Task 2 (C and D) as a function of selected response in Experiment 3. Mir, mirrored mapping; Mix, mixed mapping.

tended to make the crossed response that was correct for the opposite mapping.

\section{Discussion}

For side positions, a consistency benefit was evident for Task 1 and Task 2 performance. RTs were shorter when both task mappings were mirrored or mixed than when the mappings for Tasks 1 and 2 differed. The consistency benefit did not interact with SOA for Task 2 and did so only slightly for Task 1 . The consistency benefit for the mirrored mapping is predicted by the emergent mapping-choice account, because the task mapping for both tasks conforms to a single rule. That the consistency benefit is evident for the mixed mapping implies that a single task-mapping rule is not a necessary condition and that a benefit can occur when the global mapping of the eight positions is less complex than that for the inconsistent mapping conditions.

The middle positions, for which the responses were opposite regardless of mapping, also showed a consistency benefit, although it was smaller than that for the side positions, for which the responses differed as a function of mapping. Thus, a consistency benefit can be obtained for positions that have the same S-R pairings for both tasks even if those $\mathrm{S}-\mathrm{R}$ pairings are not all corresponding. As for the side positions, this consistency benefit is not pre- dicted for the mixed mapping if it is assumed that a single mapping rule needs to be applicable to each task.

An obvious question is why the mixed-mixed condition in Experiment 3 showed an RT consistency benefit, but that in Experiment 2 did not. This question can be answered by comparing the consistent mapping conditions of Experiments 2 and 3. As is illustrated in Figure 12, RTs were much shorter for the corresponding-corresponding condition than for the other three consistent conditions, which showed little difference. This comparison illustrates the previously noted facilitation that results from all individual S-R pairings being corresponding, which was not as obvious in the analyses of Experiment 2 alone. The lack of difference between the mixed-mixed condition of Experiment 2 , for which the middle positions were corresponding and the side positions were opposite, and the mixed-mixed condition of Experiment 3, for which these relations were reversed, indicates that these two versions of mixed mappings yield similar results. That these two mixed-mixed conditions also yielded results similar to those for the mirrored-mirrored condition indicates that there is no additional benefit of conforming to a single rule.

This comparison shows that the consistency benefit for the mixed-mixed condition (and that for the mirroredmirrored condition) in Experiment 3 was not a conse- 

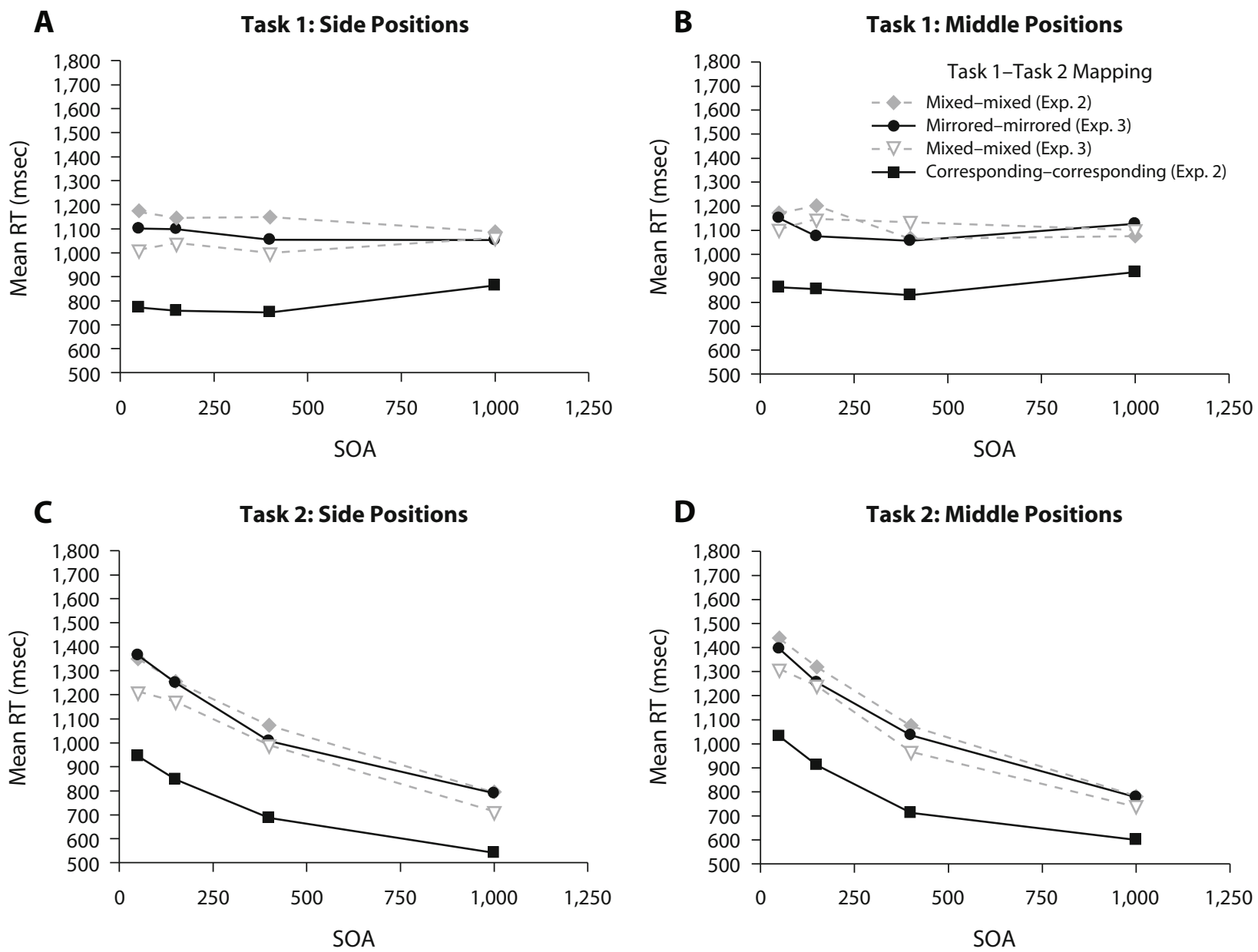

D

Task 2: Middle Positions

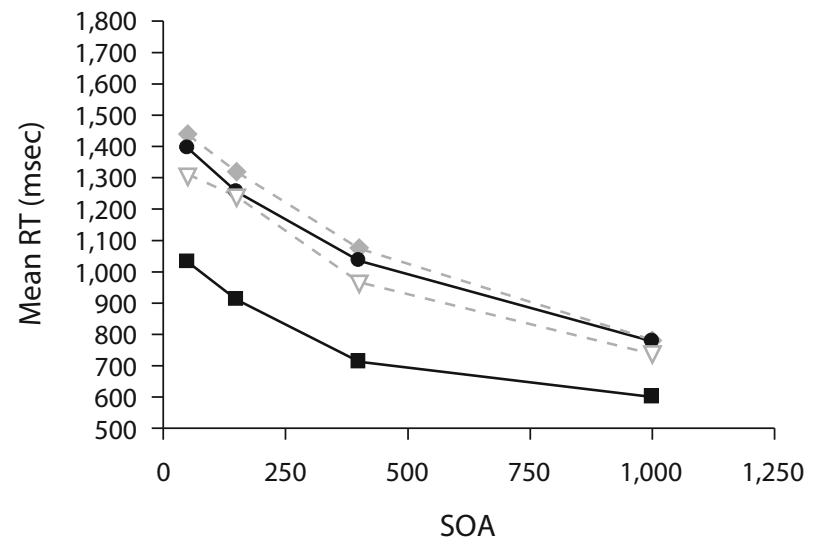

Figure 12. Mean reaction times (RTs) in Task 1 (A and B) and Task 2 (C and D) in milliseconds for the side (A and C) and middle (B and D) positions of the consistent mapping conditions in Experiments (Exp.) 2 and 3 as a function of stimulus onset asynchrony (SOA) and Task 1-Task 2 mapping.

quence of performance being facilitated by having consistent mappings but, rather, of performance suffering more from the inconsistent mappings. That is, the task combinations of mirrored-mixed and mixed-mirrored were particularly difficult for participants, as indicated by both long RTs and high PEs. In fact, the corresponding-mixed and mixed-corresponding conditions in Experiment 2 yielded RT levels similar to those for the mixed-mixed and mirrored-mirrored conditions in Experiment 3.

Why were the mirrored-mixed and mixed-mirrored conditions of Experiment 3 more difficult than the corresponding-mixed and mixed-corresponding conditions of Experiment 2? As described earlier, when one mapping is completely corresponding and the other partially corresponding, the corresponding response is required for six positions. Thus, participants can code the two tasks as a single task conforming to the rule "make the corresponding response," with an exception of "respond opposite" to the single pair of side stimulus positions that are exceptions. Such a strategy is not viable when one of the mappings is completely crossed and the other partially crossed, because the six crossed positions cannot be coded as following a single "respond opposite" rule that holds across tasks. Consequently, for the mirrored-mixed and mixed-mirrored conditions, responses cannot be selected by application of a single rule to six positions plus an exception for the other two positions.

\section{GENERAL DISCUSSION}

It is possible to reduce the costs of dual-task performance by using mappings that are spatially consistent for the two tasks, rather than inconsistent. Duncan (1979) illustrated this consistency benefit for three-choice tasks, and we replicated it for two-choice tasks (Vu \& Proctor, 2006). Although both three- and two-choice tasks show a benefit for using the same mapping, its processing basis in the two situations seems to be different. For two-choice tasks, the consistency benefit is primarily a consequence of an emergent perceptual feature that is present when two visual stimuli occur in temporal proximity. This conclusion is indicated by the fact that the benefit is largely eliminated at long SOAs and is not obtained when the stimuli are auditory for one task and visual for the other. For three-choice 
tasks, on the other hand, the consistency benefit is primarily due to an emergent mapping choice for the inconsistent mapping conditions and is evident at short and long SOAs for both Tasks 1 and 2 (Proctor \& Vu, 2008).

With three-choice tasks, the consistency benefit for an opposite mapping is obtained only at the side positions, for which the responses differ for corresponding and mirrored mappings (Proctor $\& \mathrm{Vu}, 2008$ ). If participants were applying a mirrored response rule to each task as a whole, then the consistency benefit should be apparent for the middle positions as well. The absence of a benefit for the middle positions implies that, when at least one of the task mappings is mirrored, participants first determine whether the stimulus is in a middle or a side position, and only if the decision is "side" do they proceed with applying the "respond opposite" rule (when the task mappings are consistent mirrored) or selecting and applying the rule (when the task mappings are inconsistent). That participants apparently do not apply a mirror-opposite rule to all positions of three-choice tasks when the mirrored mapping is in effect may be a consequence of the fact that the middle position requires a corresponding response rather than a crossed response. Thus, it may be more natural to prepare the corresponding response for the middle position and treat the middle-side distinction as a "middle versus rule" signal, applying the opposite rule only if the stimulus is identified as being in a side position. If participants indeed perform pairs of three-choice tasks by first determining whether the position is middle or side and then applying (or selecting and applying) the opposite rule only if the decision is "side," it should be possible to create situations for four-choice tasks that introduce a consistency benefit for a noncorresponding task mapping at the middle positions (as well as at the side positions) or to eliminate the consistency benefit for all positions, including the side positions.

For four-choice tasks, responses are crossed for both the middle and the side positions with a mirrored mapping. Because a single "respond opposite" rule truly applies to all positions with this mapping, the emergent mapping-choice account predicts that the mirrored mapping should show consistency benefits for the middle positions, as well as for the side positions, relative to when it is paired with a corresponding task mapping. Benefits for the consistent corresponding and mirrored conditions of the four-choice tasks in Experiment 1 were indeed evident for both the middle and the side positions. This result indicates that it is not simply the relative salience of the side positions (e.g., Adam et al., 1998) that leads to a consistency benefit for the mirrored mapping. Consistency benefits occurred at all SOAs, which is also in agreement with the emergent mapping-choice account, although the decrease in RT2 as SOA increased was slightly smaller for the corresponding-corresponding condition than for the other three conditions.

Another emergent property of dual-task performance is $\mathrm{R}-\mathrm{R}$ compatibility effects. These cross-task correspondence effects can arise from corresponding response locations for the two tasks or from corresponding anatomical effectors (which, in the present study, are confounded with mirroropposite locations; Hazeltine, 2005). Experiment 1 showed little evidence of $\mathrm{R}-\mathrm{R}$ compatibility effects based on the middle-side relation, suggesting that, unlike three-choice tasks, participants were not making an initial middle-side choice. This difference in findings for four- and three-choice mirrored mappings implies that the opposite rule is coded as applying to all positions for the four-choice task but only to the two crossed side positions for the three-choice task. Finally, overall, the consistency benefit was much larger for the corresponding mapping than for the mirrored mapping, providing additional evidence of facilitation that occurs as a consequence of knowing that the corresponding response will be correct in all cases.

A four-choice mixed mapping for which the responses for the side positions are crossed and those for the middle positions are corresponding, when paired with a completely corresponding mapping, has a structure similar to that of the three-choice tasks, in that only the side positions are crossed. The difference is that two middle positions, rather than one, have a corresponding mapping for the four-choice task. The emergent mapping-choice account suggests that this distinction may be crucial, because, in the four-choice situation, the mapping does not conform to a single "respond opposite" rule, which necessitates a choice between mapping rules even when the mixed mapping applies to both tasks. If the benefit of a consistent mapping arises from not needing to make a mapping choice between tasks, a benefit for consistent mixed mappings would be expected. However, if the consistent mappings for the two tasks must themselves conform to a single rule, no benefit for the mixed mapping should be found for either middle or side positions. The results of Experiment 2 showed the latter outcome, providing evidence consistent with the version of the emergent mapping-choice account that places emphasis on each task's conforming to the same rule.

For the mixed mapping used in Experiment 2, the R-R compatibility data showed an indication that a middle-side decision was being made as part of the response-selection process, as it was for three-choice tasks. Thus, this strategy seems to be employed when the middle positions have a corresponding relation. The lack of a consistency benefit for the side positions, as well as for the middle positions, with this mixed four-choice mapping seems to indicate that the initial choice between middle and side positions does not include specification of the mapping. If the stimulus is in a side position when the same mixed mapping holds for both tasks, a decision between crossed and corresponding must still be made. The completely corresponding mapping did show a consistency benefit in Experiment 2, which would again be expected on the basis of the separate facilitation component that is unique to this mapping.

If, in fact, the two task mappings must conform to a single rule for a consistency benefit to be obtained, then, when a mirrored mapping is paired with a mixed mapping for which the side positions are corresponding and the middle ones crossed, only the mirrored mapping should show a consistency benefit. As for Experiment 2, this prediction is made because the mixed mapping does not adhere to a single mapping rule, and a choice between 
rules is necessary even when that mapping is in effect for both tasks. To the contrary, Experiment 3 showed consistency benefits for both the mirrored and mixed mappings. Comparison with Experiment 2 indicated that this consistency benefit was due to the inconsistent conditions of Experiment 3-for which one mapping was mirrored and the other mixed - being much more difficult than the inconsistent conditions of Experiment 2-for which one mapping was corresponding and the other mixed.

As was described earlier, the difference in difficulty of the inconsistent mapping conditions in the two experiments seems to be a consequence of whether or not the mapping rule for the majority of positions can be applied to the positions of the two tasks as a whole. When six of the eight positions conform to the corresponding rule, as they did in Experiment 2, the rule can be applied to those six positions without regard to whether a particular position is in Task 1 or Task 2. However, when six of the eight positions follow the mirror-opposite rule, as they did in Experiment 3, this rule must be applied separately to each task. Consequently, in the former case, a strategy can be adopted of making the corresponding response unless the stimulus is in one of the two positions that do not conform to the rule. But, in the latter case, a similar strategy cannot be used. When one mapping is mirrored and the other is mixed, at least three rules are applicable: an opposite rule for one task and, for the other task, a mirror-opposite mapping for one pair of stimulus positions and a corresponding mapping for the other pair. Thus, although the results of Experiment 3 are not predicted by the emergent mapping-choice account if the emphasis is solely on the individual task mappings, they are in agreement with Duncan's (1979) more general point that emergent properties of the tasks in combination are key determinants of dual-task performance.

An interesting finding across Experiments $1-3$ is that, when a mirrored mapping was paired with another mapping, the errors were more frequent on the task without the mirrored mapping. This outcome occurred regardless of whether the mirrored mapping was used for Task 1 or for Task 2 and was clearly evident even when the alternative mapping was corresponding (Experiments 1 and 2). The latter finding is counterintuitive, because the corresponding response is typically the more natural response tendency, indicated, for example, by the fact that corresponding errors when both tasks use an opposite mapping are more frequent than opposite errors when both tasks use a corresponding mapping. The predominance of opposite errors when the task mappings differ implies that the mirrored mapping is kept relatively more active in working memory than is the alternative mapping.

In the dual-task studies to date in which mappings have been varied for pairs of spatial choice tasks performed with keypresses executed by fingers on each hand, performance has been best when a consistent corresponding mapping is used for both tasks. Hazeltine (2005) found an exception for mappings of numeric stimuli to keypress pairs in four-choice tasks. He mapped the digits 1, 2, 3, and 4 in a left-right compatible order to responses for some participants (left-to-right order for both left-hand and right-hand keypresses) and in an anatomically com- patible order for others $(4,3,2,1$ left-to-right order for the left hand and 1, 2, 3, 4 for the right hand). Performance was better with the anatomically compatible mapping than with the left-right compatible mapping, which violates a consistency benefit in terms of spatial assignments to keypresses. The anatomically consistent (but spatially inconsistent) numeric mappings in Hazeltine's study differ from the spatially inconsistent mappings of the present study in that the incompatible 4, 3, 2, 1 left-to-right mapping does not require crossed responses, whereas the mirrored spatial mapping does. This consistent ordering may allow participants to benefit from the anatomical correspondence when it is paired with a 1, 2, 3, 4 left-to-right mapping for the right hand.

Hazeltine (2005) also included intermixed trials, similar to those of the present study, in which the stimulus for one or both tasks was a spatial location that corresponded to the response to be made. RTs for these trials varied as a function of the digit mappings in a manner similar to the variation in the digit trials, indicating that participants were not responding automatically to the stimulus location. In fact, RTs to each stimulus for trials on which both stimuli were spatial locations averaged more than $1 \mathrm{sec}$. This outcome is in agreement with the general finding that mixing mappings, whether in dual-task contexts, as in the present study, or in single-task contexts (Shaffer, 1965; Vu \& Proctor, 2004), increases overall RTs and reduces or eliminates the benefit of spatial correspondence.

In conclusion, Experiments 1 and 2 confirmed opposing predictions of the emergent mapping-choice account with different combinations of task mappings: When a mirrored mapping was paired with a corresponding mapping, it showed a consistency benefit for the middle positions, as well as for the side positions; when a mixed mapping (for which only the side positions were crossed) was paired with a corresponding mapping, there was no consistency benefit for the middle or side positions. The results of Experiments 2 and 3 provide evidence of another emergent property, that of whether a rule can be applied across tasks or whether the rule must be applied separately within each task. The experiments also confirm that an additional component operates when the mappings for both tasks are completely corresponding: Facilitation arises from knowing in advance that the corresponding response is always the correct one. For two-choice tasks, another determinant of the consistency benefit is an emergent perceptual feature of blank versus filled locations that allows corresponding responses for the two tasks as a whole to be made even when both task mappings are opposite. The results of the present study converge with those of previous ones in supporting Duncan's (1979) general point that an emergent mapping choice "is only one of many possible emergent processes" (p. 216) that can affect the efficiency of response selection in dual-task contexts.

\section{AUTHOR NOTE}

This study was supported in part by Grant W911NF-05-1-0153 from the Army Research Office to R.W.P. and by a CSULB Psychology Department 22B Research Award to K.-P.L.V. We thank John Duncan, Eliot Hazeltine, and Carlo Umiltà for their comments on the manuscript. Correspondence 
concerning this article should be addressed to R. W. Proctor, Department of Psychological Sciences, Purdue University, 703 Third Street, W. Lafayette, IN 47907-2081 (e-mail: proctor@psych.purdue.edu).

\section{REFERENCES}

Adam, J. J., Paas, F. G. W. C., Teeken, J. C., van Loon, E. M., van Boxtel, M. P. J., Houx, R. J., \& Umiltà, C. (1998). Effects of age on performance in a finger-precuing task. Journal of Experimental Psychology: Human Perception \& Performance, 24, 870-883.

Albert, N. B., Weigelt, M., Hazeltine, E., \& Ivry, R. B. (2007). Target selection during bimanual reaching to direct cues is unaffected by the perceptual similarity of the targets. Journal of Experimental Psychology: Human Perception \& Performance, 33, 1107-1116.

Duncan, J. (1977). Response selection rules in spatial choice reaction tasks. In S. Dornic (Ed.), Attention and performance VI (pp. 49-61). Hillsdale, NJ: Erlbaum.

Duncan, J. (1979). Divided attention: The whole is more than the sum of its parts. Journal of Experimental Psychology: Human Perception \& Performance, 5, 216-228.

Ehrenstein, A., \& Proctor, R. W. (1998). Selecting mapping rules and responses in mixed compatibility four-choice tasks. Psychological Research, 61, 231-248.

HaZeltine, E. (2005). Response-response compatibility during bimanual movements: Evidence for the conceptual coding of action. Psychonomic Bulletin \& Review, 12, 682-688.

Ivry, R. B., Franz, E. A., Kingstone, A., \& Johnston, J. C. (1998). The psychological refractory period effect following callosotomy: Uncoupling of lateralized response codes. Journal of Experimental Psychology: Human Perception \& Performance, 24, 463-480.

Lien, M.-C., \& Proctor, R. W. (2000). Multiple spatial correspondence effects on dual-task performance. Journal of Experimental Psychology: Human Perception \& Performance, 26, 1260-1280.
PAshler, H. (1994). Dual task interference in simple tasks: Data and theory. Psychological Bulletin, 2, 220-244.

Proctor, R. W., \& Vu, K.-P. L. (2008). Determinants of the benefit for consistent stimulus-response mappings in dual-task performance of three-choice tasks. Manuscript submitted for publication.

Proctor, R. W., Vu, K-.P. L., \& PicK, D. F. (2006). A deficit in effortful selection of cued responses for older adults. Journal of Motor Behavior, 38, 265-284.

SchUCH, S., \& KосH, I. (2004). The costs of changing the representation of action: Response repetition and response-response compatibility in dual tasks. Journal of Experimental Psychology: Human Perception \& Performance, 30, 566-582.

Shaffer, L. H. (1965). Choice reaction with variable S-R mapping. Journal of Experimental Psychology, 70, 284-288.

Strayer, D. L., Drews, F. A., \& Johnston, W. A. (2003). Cell phone induced failures of visual attention during simulated driving. Journal of Experimental Psychology: Applied, 9, 23-32.

STOFFELS, E.-J. (1996). Uncertainty and processing routes in the selection of a response: An S-R compatibility study. Acta Psychologica, 94, 227-252.

VU, K.-P. L., \& Proctor, R. W. (2004). Mixing compatible and incompatible mappings: Elimination, reduction, and enhancement of spatial compatibility effects. Quarterly Journal of Experimental Psychology, 57A, 539-556.

Vu, K.-P. L., \& Proctor, R. W. (2006). Emergent perceptual features in the benefit of consistent stimulus-response mappings on dual-task performance. Psychological Research, 70, 468-483.

YAMAgUChI, M., \& Proctor, R. W. (2006). Stimulus-response compatibility with pure and mixed mappings in a flight task environment. Journal of Experimental Psychology: Applied, 12, 207-222.

Zhang, J., Patel, V. L., Johnson, T. R., \& Shortliffe, E. H. (2004). A cognitive taxonomy of medical errors. Journal of Biomedical Informatics, 37, 193-204.

\begin{abstract}
APPENDIX A
R-R Compatibility Analysis

\section{Experiment 1}

An ANOVA of RT1 as a function of position (whether R1 and S1 were in middle or side positions) and R-R compatibility (whether R2 was in the position compatible with that of R1, the mirror-opposite position from $\mathrm{R} 1$, or one of the incompatible positions assigned to the alternate pair of locations [e.g., if R1 was at a side, R2 was in the middle]) was performed. Task 1 mapping and Task 2 mapping were also included as factors. None of the terms involving R-R compatibility was significant, indicating that R2 (and S2) had little influence on RT1 (see Figures $3 \mathrm{~A}$ and $3 \mathrm{~B}$ ).

A similar analysis of RT2 (see Figures $3 \mathrm{C}$ and 3D) showed a main effect of R-R compatibility $[F(2,30)=$ $6.77, p=.006]$ : RT2 was shorter when R1 was compatible $(M=1,013 \mathrm{msec})$ or mirrored $(M=1,015 \mathrm{msec})$ than when it was incompatible $(M=1,046 \mathrm{msec})$. R-R compatibility interacted with Task 2 mapping $[F(2,30)=$ $3.60, p=.046]$, and this was qualified by a three-way interaction of those variables with Task 1 mapping $[F(2,30)=5.03, p=.014]$. When the mappings were inconsistent, there were no R-R compatibility effects on RT2 $(F \mathrm{~s}<1)$. For consistent corresponding mappings, there was an R-R compatibility effect $[F(2,30)=8.19$, $p=.003]$. Bonferroni follow-up tests showed that the only reliable difference was between the mirror-opposite relation $(M=740 \mathrm{msec})$ and the incompatible relation $(M=797 \mathrm{msec})$, with the compatible relation $(M=$ $780 \mathrm{msec}$ ) not being significantly different from the other two. For consistent mirrored mappings, there was also an R-R compatibility effect $[F(2,30)=17.73, p<.001]$. Bonferroni tests showed no difference between compatible $(M=974 \mathrm{msec})$ and mirror-opposite $(M=1,001 \mathrm{msec})$ relations, with RTs being longer when the relation was incompatible $(M=1,074 \mathrm{msec})$.

$\mathrm{R}-\mathrm{R}$ compatibility for RT2 also interacted with the middle versus side distinction $[F(2,30)=10.20, p=$ $.001]$. The side positions showed an $\mathrm{R}-\mathrm{R}$ compatibility effect $[F(2,30)=16.97, p<.001]$, for which the compatible $(M=951 \mathrm{msec})$ and mirror-opposite $(M=970 \mathrm{msec})$ relations did not differ and were faster than the incompatible relation $(M=1,046 \mathrm{msec})$. However, the middle positions did not show a significant $\mathrm{R}-\mathrm{R}$ compatibility effect $(M \mathrm{~s}=1,075,1,060$, and $1,045 \mathrm{msec}$, respectively) $[F(2,30)=1.49, p=.242]$. The two-way interaction of $\mathrm{R}-\mathrm{R}$ compatibility with middle versus side position was qualified by a three-way interaction of those variables with Task 1 mapping $[F(2,30)=6.27, p=.005]$. When the Task 1 mapping was corresponding, $\mathrm{R}-\mathrm{R}$ compatibility effects were obtained for both the side and the middle positions $\left[F_{\mathrm{S}}(2,30)>5.88, p \mathrm{~s}<.007\right]$ : For side positions, the compatible and mirror-opposite conditions were significantly faster than the incompatible condition ( $M \mathrm{~s}=881,882$, and $978 \mathrm{msec}$, respectively), but, for middle positions, this pattern was reversed
\end{abstract}




\section{APPENDIX A (Continued)}

( $M \mathrm{~s}=1,029,1,008$, and $952 \mathrm{msec}$, respectively). When the mapping was mirrored, an R-R compatibility effect was obtained only for the side positions $[F(2,30)=6.94, p=.008]$ : Compatible RT2 $(M=1,022 \mathrm{msec})$ was shorter than incompatible RT2 $(M=1,115 \mathrm{msec})$, and the mirror-opposite condition $(M=1059 \mathrm{msec})$ did not differ significantly from either of those.

\section{Experiment 2}

R-R compatibility was analyzed for RT1 and RT2, similar to the analyses in Experiment 1 . For RT1 (see Figures 7A and 7B), the main effect of $\mathrm{R}-\mathrm{R}$ compatibility was significant $[F(2,30)=6.48, p=.005]$. RT1 tended to be shorter for the side positions when R2 was compatible $(M=1,013 \mathrm{msec})$ or mirror opposite $(M=$ $1,001 \mathrm{msec})$ than when it was incompatible $(M=1,036 \mathrm{msec})$, with only the latter difference being significant. The three-way interaction of $\mathrm{R}-\mathrm{R}$ compatibility with Task 1 mapping and middle versus side position was significant $[F(2,30)=6.12, p=.009]$ : An $\mathrm{R}-\mathrm{R}$ compatibility effect was evident only for the combination of mixed mapping and middle position $[F(2,30)=9.46, p<.001]$. RT1 was similar for the compatible $(M=$ $1,039 \mathrm{msec})$ and mirror-opposite $(M=1,021 \mathrm{msec})$ relations and longer for the incompatible relation $(M=$ $1,107 \mathrm{msec}$ ). This three-way interaction was qualified by a four-way interaction of those variables with Task 2 mapping $[F(2,30)=6.24, p=.005]$, in which this pattern was evident primarily when the Task 2 mapping was corresponding $[F(2,30)=17.58, p=.001]$ (see Figures 7A and 7B).

The main effect of R-R compatibility was also significant for RT2 (see Figures $7 \mathrm{C}$ and $7 \mathrm{D})[F(2,30)=32.50$, $p<.001]$. The result pattern was similar to that for RT1 but more pronounced: RT2 did not differ significantly when R1 position was compatible $(M=996 \mathrm{msec})$ relative to when it was mirror opposite $(M=971 \mathrm{msec})$, but both of those conditions differed from the incompatible condition $(M=1,059 \mathrm{msec})$. R-R compatibility entered into several significant interactions: Two-way interactions with Task 1 mapping $[F(2,30)=6.78, p<$ $.004]$ and with middle versus side position $[F(2,30)=5.79, p<.008]$, a three-way interaction with Task 1 mapping and Task 2 mapping $[F(2,30)=3.62, p<.043]$, and a four-way interaction of all variables $[F(2,30)=$ $22.70, p<.001]$ (see Figures 7C and 7D). For the corresponding-corresponding task mappings, $\mathrm{R}-\mathrm{R}$ compatibility effects were evident only for the side positions $[F(2,30)=16.95, p<.001]$. As for the main effect, there was no difference between when R1 position was compatible $(M=689 \mathrm{msec})$ and when it was mirror opposite $(M=682 \mathrm{msec})$, but both of those conditions differed from the incompatible condition $(M=825 \mathrm{msec})$. For the corresponding-mixed condition, $\mathrm{R}-\mathrm{R}$ compatibility effects were evident for the middle positions $[F(2,30)=$ $15.19, p<.001]$ : There was again no difference between when R1 position was compatible $(M=979 \mathrm{msec})$ and when it was mirror opposite $(M=951 \mathrm{msec})$, but both of those conditions differed from the incompatible condition $(M=1,129 \mathrm{msec})$. For the mixed-corresponding condition, R-R compatibility effects were evident for the middle positions $[F(2,30)=22.06, p<.001]$ : There was again no difference between when $\mathrm{R} 1$ position was compatible $(M=906 \mathrm{msec})$ and when it was mirror opposite $(M=914 \mathrm{msec})$, but both of those conditions differed from the incompatible condition $(M=1,112 \mathrm{msec})$. For the mixed-mixed condition, $\mathrm{R}-\mathrm{R}$ compatibility effects were evident at both middle and side positions $[F \mathrm{~s}(2,30)>6.79, p \mathrm{~s}<.008]$ : For both middle and side positions, the mirror-opposite condition $(M \mathrm{~s}=1,056$ and $1,001 \mathrm{msec}$, respectively) was faster than the incompatible condition ( $M=1,210$ and $1,172 \mathrm{msec}$, respectively), and the compatible condition $(M=1,140$ and $1,132 \mathrm{msec}$, respectively) did not differ significantly from either.

\section{Experiment 3}

For RT1 (see Figures 10A and 10B), there was a main effect of R-R compatibility $[F(2,30)=5.07, p<$ .013]. RT1 tended to be shorter for the side positions when R2 was compatible ( $M=1,183 \mathrm{msec})$ or mirror opposite $(M=1,164 \mathrm{msec})$ than when it was incompatible $(M=1,203 \mathrm{msec})$, but only the latter difference was significant. The three-way interaction of R-R compatibility with Task 1 mapping and Task 2 mapping was significant $[F(2,30)=3.41, p=.046]$, as was the four-way interaction of those variables with middle versus side position $[F(2,30)=5.77, p=.008]$. $\mathrm{R}-\mathrm{R}$ compatibility effects were evident for the consistent mapping conditions $\left[F_{\mathrm{S}}(2,30)>4.01, p \mathrm{~s}=.030\right]$ but not for the inconsistent ones $\left(F_{\mathrm{S}}<1\right)$ (see Figures 10A and 10B). For the mirrored-mirrored mapping condition, RT2 was significantly shorter for the mirror-opposite relation $(M=1,065 \mathrm{msec})$ than for the incompatible relation $(M=1,132 \mathrm{msec})$, with the compatible relation $(M=$ $1,107 \mathrm{msec}$ ) being intermediate and not significantly different from either. For the mixed-mixed mapping condition, the pattern was similar $(M \mathrm{~s}=1,048,1,122$, and $1,098 \mathrm{msec}$, respectively), although no post hoc comparison attained the .05 level. The four-way interaction was due to the pattern for the mirrored-mirrored condition holding mainly for the middle positions and that for the mixed-mixed condition holding mainly for the side positions.

For RT2 (see Figures 10C and 10D), R-R compatibility showed a main effect $[F(2,30)=38.62, p<.001]$. RT2 was shorter when the relation was mirror opposite $(M=1,132 \mathrm{msec})$ than when it was compatible $(M=$ $1,188 \mathrm{msec})$, which in turn was shorter than when it was incompatible $(M=1,253 \mathrm{msec})$. The three-way interaction of $\mathrm{R}-\mathrm{R}$ compatibility with Task 1 mapping and Task 2 mapping $[F(2,30)=3.61, p=.039]$ and that with Task 1 mapping and middle-side position $[F(2,30)=5.98, p=.008]$ were significant, as was the four-way interaction of all variables $[F(2,30)=13.73, p<.001]$. For the mirrored-mirrored task mappings, $\mathrm{R}-\mathrm{R}$ compatibility effects were evident only for middle and side positions $\left[F_{\mathrm{S}}(2,30)>5.33, p<.013\right]$ : For both positions, the only significant difference was between the mirror-opposite $(M \mathrm{~s}=1,031$ and $1,043 \mathrm{msec}$, respectively) and the incompatible $(M \mathrm{~s}=1,196$ and 1,190 $\mathrm{msec}$, respectively) conditions, with the compatible condition being intermediate 


\section{APPENDIX A (Continued)}

( $M \mathrm{~s}=1,129$ and $1,113 \mathrm{msec}$, respectively). For the mirrored-mixed condition, $\mathrm{R}-\mathrm{R}$ compatibility effects were significant only for the middle positions $[F(2,30)=10.61, p<.001]$ : RT2 did not differ significantly between when R1 position was mirror opposite $(M=1,139 \mathrm{msec})$ and when it was compatible $(M=1,171 \mathrm{msec})$, but both were shorter than when it was incompatible $(M=1,373 \mathrm{msec})$. For the mixed-mirrored condition, $\mathrm{R}-\mathrm{R}$ compatibility effects were evident for the middle positions $[F(2,30)=6.07, p=.026]$ : There was again no difference between when R1 position was compatible $(M=1,185 \mathrm{msec})$ and when it was mirror opposite $(M=1,165 \mathrm{msec})$, and the latter differed from the incompatible condition $(M=1,309 \mathrm{msec})$. For the mixed-mixed condition, $\mathrm{R}-\mathrm{R}$ compatibility effects were evident at the side positions $[F(2,30)=30.11, p<.001]$ : The compatible $(M=$ $963 \mathrm{msec})$ and mirror-opposite $(M=878 \mathrm{msec})$ relations did not differ from each other, and both yielded shorter RT2s than did the incompatible condition ( $M=1,146 \mathrm{msec}$; see Figures $10 \mathrm{C}$ and $10 \mathrm{D})$.

\section{APPENDIX B}

Error Type Analyses

\section{Experiment 1}

For the consistent mapping conditions, an incorrect response could be in the mirror-opposite position, an adjacent position (non-mirror-opposite for the middle responses), or another position (neither adjacent nor mirror opposite). For the inconsistent conditions, the mirror-opposite response was also the one that would be correct for the alternative mapping rule.

For Task 1 side positions (see Figure 4A), there was a difference in error patterns for the two consistent conditions $\left[\chi^{2}(2)=5.32, p=.021\right]$ : Only adjacent and mirror-opposite errors were made; for the correspondingcorresponding condition, adjacent errors tended to outnumber mirror-opposite errors $\left[\chi^{2}(1)=3.76, p=.052\right]$, whereas there was no significant difference in frequency of the two error types for the opposite-opposite condition $\left[\chi^{2}(1)=1.67, p=.197\right]$. Similarly, there was no difference in error patterns for the two inconsistent mapping conditions $\left[\chi^{2}(2)=2.04, p=.36\right]$ : But in this case, more alternative-rule errors were committed than adjacent and other errors $\left[\chi^{2}(2)=160.2, p<.001\right]$. For Task 1 middle positions (see Figure 4B), there was no significant difference in error patterns for the two consistent conditions $\left[\chi^{2}(2)=5.33, p=.07\right]$ : There were more errors involving the mirror-opposite position than there were either of the other two error types $\left[\chi^{2}(2)=38.78, p<\right.$ $.001]$. Also, there was no difference in error patterns for the two inconsistent conditions $\left[\chi^{2}(2)<1\right]$ : For these conditions, more alternative-rule errors were made than adjacent and other errors $\left[\chi^{2}(2)=233.77, p<.001\right]$.

Unlike for Task 1, for Task 2 side positions (see Figure 4C), there was a significant difference in error patterns for the consistent mapping conditions $\left[\chi^{2}(2)=7.05, p=.029\right]$ : For the corresponding-corresponding condition, adjacent errors outnumbered mirror-opposite and other errors $\left[\chi^{2}(2)=37.02, p<.001\right]$, whereas, for the mirrored-mirrored condition, errors were divided relatively equally between the adjacent and mirror-opposite types, with other errors being less frequent. There was also a significant difference in error patterns for the inconsistent conditions $\left[\chi^{2}(2)=12.85, p=.002\right]$ : For the corresponding-mirrored condition, alternative-rule errors outnumbered adjacent and other errors $\left[\chi^{2}(2)=68.53, p<.001\right]$; for the mirrored-corresponding condition, even more alternative-rule errors were made, and the remaining errors were all adjacent $\left[\chi^{2}(2)=74.60\right.$, $p<.001]$. For Task 2 middle positions (see Figure 4D), there was no significant difference in error patterns for the consistent mapping conditions $\left[\chi^{2}(2)=3.72, p=.16\right]$ : Errors were frequent for the adjacent middle position and the adjacent side position but not for the other side position $\left[\chi^{2}(2)=76.62, p<.001\right]$. There was also no difference in error patterns for the inconsistent mapping conditions $\left[\chi^{2}(2)<1\right]$ : More alternative-rule errors were committed than adjacent and other errors $\left[\chi^{2}(2)=276.01, p<.001\right]$. Thus, with inconsistent mappings, the majority of errors were ones that were in agreement with the alternative mapping rule.

\section{Experiment 2}

For Task 1 side positions, there was a significant difference in error patterns for the two consistent mapping conditions (see Figure 8 A) $\left[\chi^{2}(2)=28.98, p<.001\right]$ : For the corresponding-corresponding condition, more adjacent errors than mirror-opposite or other errors were made $\left[\chi^{2}(2)=20.33, p<.001\right]$; for the mixed-mixed condition, there were more mirror-opposite errors (i.e., making the corresponding response instead of the one opposite to the stimulus location) than adjacent errors $\left[\chi^{2}(2)=18.94, p<.001\right]$. For the two inconsistent mapping conditions, there was a significant difference in error patterns $\left[\chi^{2}(2)=15.67, p<.001\right]$ : For the corresponding-mixed condition, more alternative-rule errors were committed than adjacent and other errors $\left[\chi^{2}(2)=81.92, p<.001\right]$. For the mixed-corresponding condition, more alternative-rule errors were committed than other errors $\left[\chi^{2}(1)=55.05, p<.001\right]$, and no adjacent errors were made. For Task 1 middle positions (see Figure $8 \mathrm{~B}$ ), there was again a significant difference in error patterns for the two consistent mapping conditions $\left[\chi^{2}(2)=7.08, p=.029\right]$ : For the corresponding-corresponding condition, the most errors involved the mirroropposite position (i.e., the alternative middle response) and the fewest errors the other position $\left[\chi^{2}(2)=13.00\right.$, $p=.002]$; for the mixed-mixed condition, the pattern of more errors for the mirror-opposite position than for the adjacent and other positions was more pronounced $\left[\chi^{2}(2)=51.05, p<.001\right]$. There was no difference in error patterns for the two inconsistent mapping conditions $\left[\chi^{2}(2)=1.43, p>.20\right]$ : For these conditions, more alternative-rule errors were made than adjacent errors $\left[\chi^{2}(2)=14.54, p<.001\right]$. 


\section{APPENDIX B (Continued)}

For Task 2 side positions (see Figure $8 \mathrm{C}$ ), there was a significant difference in error patterns for the consistent mapping conditions $\left[\chi^{2}(2)=67.15, p<.001\right]$ : For the corresponding-corresponding condition, adjacent errors outnumbered mirror-opposite and other errors $\left[\chi^{2}(2)=44.36, p<.001\right]$, whereas, for the mixed-mixed condition, more mirror-opposite errors were committed than adjacent and other errors $\left[\chi^{2}(2)=63.97, p<.001\right]$. There was no significant difference in error patterns for the inconsistent conditions $\left[\chi^{2}(2)=3.99, p>.13\right]$ : Alternative-rule errors were more frequent than adjacent and other errors $\left[\chi^{2}(2)=263.72, p<.001\right]$. For Task 2 middle positions (see Figure 8D), there was a significant difference in error patterns for the consistent mapping conditions $\left[\chi^{2}(2)=13.65, p<.001\right]$ : For the corresponding-corresponding condition, errors were evenly distributed between the mirror-opposite and adjacent positions and outnumbered those for the other position $\left[\chi^{2}(2)=23.74, p<.001\right]$. For the mixed-mixed condition, more mirror-opposite errors were made than adjacent and other errors $\left[\chi^{2}(2)=40.76, p<.001\right]$. There was also a difference in error patterns for the inconsistent mapping conditions $\left[\chi^{2}(2)=33.13, p<.001\right]$ : For the corresponding-mixed condition, more alternative-rule errors were committed than adjacent and other errors $\left[\chi^{2}(2)=90.25, p<.001\right]$. For the mixed-corresponding condition, similar numbers of alternative-rule and adjacent errors were made $\left[\chi^{2}(2)<1\right]$.

To summarize, for the inconsistent mapping conditions, the results are in agreement with the view that errors tend to be the response that would be correct if the alternative mapping were in effect. For the consistent corresponding condition, most of the errors for side positions were adjacent responses, and, for the middle positions, the errors were distributed similarly between mirror-opposite and adjacent errors, both of which involved pressing an adjacent key. However, for the consistent mixed condition, most of the errors were the mirror-opposite response, which would not be the correct response if the mapping for the wrong task were selected, because the tasks used the same mixed mapping, but would be correct if the alternative individual submapping were in effect. Thus, the error analysis shows that, with the mixed mapping, there is a cost associated with an emergent choice between mapping rules within the task, but not between a task using the mixed mapping and one using a corresponding mapping.

\section{Experiment 3}

For Task 1 side positions (see Figure 11A), there was no significant difference in error patterns for the two consistent mapping conditions $\left[\chi^{2}(2)<1\right]$ : More mirror-opposite errors were made than adjacent and other errors $\left[\chi^{2}(2)=45.70, p<.001\right]$. For the two inconsistent mapping conditions, there was also no significant difference in the error patterns $\left[\chi^{2}(2)=2.74, p>.25\right]$ : More alternative-rule errors were committed than adjacent and other errors $\left[\chi^{2}(2)=257.82, p<.001\right]$. For Task 1 middle positions (see Figure 11B), there was a significant difference in error patterns for the two consistent mapping conditions $\left[\chi^{2}(2)=10.75, p=.005\right]$ : For the mirrored-mirrored condition, there was no difference between mirror-opposite errors and adjacent errors $\left(\chi^{2}<1\right)$; for the mixed-mixed condition, more errors involved the mirror-opposite position than the adjacent and other positions $\left[\chi^{2}(2)=15.38, p<.001\right]$. There was no difference in error patterns for the two inconsistent mapping conditions $\left[\chi^{2}(2)=1.56, p>.20\right]$ : More alternative-rule errors were made than adjacent errors $\left[\chi^{2}(1)=9.00, p=.003\right]$.

For Task 2 side positions (see Figure 11C), there was a difference in error patterns for the consistent mapping conditions $\left[\chi^{2}(2)=10.43, p=.005\right]$ : For the mirrored-mirrored condition, mirror-opposite and adjacent errors outnumbered other errors $\left[\chi^{2}(2)=11.20, p=.004\right]$, whereas, for the mixed-mixed condition, mirror-opposite and other errors outnumbered adjacent errors $\left[\chi^{2}(2)=6.26, p=.044\right]$. There was a difference in error patterns for the inconsistent conditions $\left[\chi^{2}(2)=9.74, p=.008\right]$ : For the mirrored-mixed condition, alternative-rule errors were more frequent than adjacent and other errors $\left[\chi^{2}(2)=239.70, p<.001\right]$; for the mixed-mirrored condition, alternative-rule errors outnumbered adjacent and other errors to a lesser extent $\left[\chi^{2}(2)=82.07, p<\right.$ $.001]$. For Task 2 middle positions (see Figure 11D), there was a significant difference in error patterns for the consistent mapping conditions $\left[\chi^{2}(2)=8.49, p=.014\right]$ : For the mirrored-mirrored condition, errors were closely distributed between the mirror-opposite and the adjacent position and outnumbered those for the other position $\left[\chi^{2}(2)=6.08, p=.048\right]$. For the mixed-mixed condition, more mirror-opposite errors were made than adjacent and other errors $\left[\chi^{2}(2)=64.38, p<.001\right]$. There was no difference in error patterns for the inconsistent mapping conditions $\left[\chi^{2}(2)=3.60, p>.016\right]$ : More alternative-rule errors were committed than adjacent and other errors $\left[\chi^{2}(2)=109.88, p<.001\right]$.

As in Experiment 2, the mixed conditions showed an intrusion of the alternative S-R mapping, even when the task mapping was consistent (i.e., the mixed-mixed condition). For the inconsistent conditions, the stable trend was for errors to be the response that would have been correct if the mapping of the alternative task were in effect.

(Manuscript received September 15, 2008;

revision accepted for publication November 26, 2008.) 